\title{
Exact likelihood inference in group interaction network models
}

\author{
Grant Hillier \\ CeMMAP and Department of Economics, University of Southampton, \\ Highfield, Southampton SO17 1BJ, UK \\ ghh@soton.ac.uk \\ Federico Martellosio \\ School of Economics, University of Surrey, \\ Guildford, Surrey, GU2 7XH, UK \\ f.martellosio@surrey.ac.uk
}

October 162016

\begin{abstract}
The paper studies spatial autoregressive models with group interaction structure, focussing on estimation and inference for the spatial autoregressive parameter $\lambda$. The quasi-maximum likelihood estimator for $\lambda$ usually cannot be written in closed form, but using an exact result obtained earlier by the authors for its distribution function, we are able to provide a complete analysis of the properties of the estimator, and exact inference that can be based on it, in models that are balanced. This is presented first for the so-called pure model, with no regression component, but is also extended to some special cases of the more general model. We then study the much more difficult case of unbalanced models, giving analogues of some, but by no means all, of the results obtained for the balanced case earlier. In both balanced and unbalanced models, results obtained for the pure model generalize immediately to the model with group-specific regression components.

Keywords: bias, group interaction model, maximum likelihood estimation, spatial autoregression.

JEL Classification: C12,C21.

Acknowledgements: We thank the co-editor, Rob Taylor, the three referees, and Peter Phillips for comments on the first version of the paper that improved its presentation considerably.
\end{abstract}




\section{Introduction}

One important application of spatial autoregressive (SAR) models is to the analysis of social networks, particularly for the case when an outcome variable is observed on a predetermined network; see, for instance Bramoullé, Djebbari and Fortin (2009), Lee, Liu and Lin (2010), and de Paula (2016). ${ }^{1}$ Consider a fixed network of $n$ individuals, represented by a $n \times n$ weights matrix $W$. The matrix $W$ could be a $(0,1)$ adjacency matrix, a rowstandardized adjacency matrix, or could more generally be specified in such a way that the entry $W_{i, j}$ reflects the influence of individual $j$ on individual $i$. A popular specification of a SAR model for the determination of an $n \times 1$ outcome vector $y$, given the network and an $n \times k$ matrix $X$ of covariates, is

$$
y=\lambda W y+X \beta+W X \delta+\sigma \varepsilon
$$

where $\lambda$ is a scalar autoregressive parameter, $\beta$ and $\delta$ are $k \times 1$ parameters, $\sigma$ is a scale parameter, and $\varepsilon$ an $n \times 1$ error term. In the peer effects literature, $\lambda$ captures the endogenous effect, and $\delta$ the exogenous effect; see Manski (1993). In addition to social networks, model (1.1) has been applied to several other cross-sectional contexts. Also, when $W$ is block-diagonal, model (1.1) can be seen as a panel data model with crosssectional dependence - see for instance the recent paper by Robinson and Rossi (2015a), and references therein. ${ }^{2}$

A fundamental, at least conceptually, specification for the matrix $W$ in the social network literature is given by the equal weights matrix $B_{n}:=(n-1)^{-1}\left(\iota_{n} \iota_{n}^{\prime}-I_{n}\right)$, where $\iota_{n}$ denotes the $n \times 1$ vector of ones. In that case, model (1.1) postulates that the outcome variable for individual $i$ is explained by the "leave-own-out" mean $(n-1)^{-1} \sum_{j \neq i} y_{j}$, the regressors, and the leave-own-out means of the regressors; see, e.g., Moffitt (2001). The weights matrix $B_{n}$ may be appropriate when all individuals are equally affected by all other individuals, or when no information on how individuals interact is available.

A more general assumption is that individuals interact in groups, with each group member being equally affected by all the other members in that group, and with no links across groups. This results in $W$ having a block diagonal structure, with equal weights matrices as blocks. More precisely, letting $m_{i}$ be the distinct group sizes, for $i=1, \ldots, p$, and $r_{i}$ the number of groups of size $m_{i}$, for $i=1, \ldots, p$, the (row-standardized) group

\footnotetext{
${ }^{1}$ For extensions of SAR models that allow for endogenous network formation, see, e.g., Hsieh and Lee (2016).

${ }^{2}$ A special case of the model in Robinson and Rossi (2015a) is discussed in Section 3.6.1 below.
} 
interaction weights matrix is

$$
W=\operatorname{diag}\left(I_{r_{i}} \otimes B_{m_{i}}, i=1, . ., p\right) .
$$

Such matrices were used, for example, in Case (1992), Baltagi (2006), Kelejian, Prucha, and Yuzefovich (2006), Lee (2007), and Davezies et al. (2009), and this is the structure we shall consider in this paper.

We focus on inference on $\lambda$, which is often the key parameter in applications, and, for simplicity (but without loss of generality), take $\delta=0$ in (1.1). We call a model

$$
y=\lambda W y+X \beta+\sigma \varepsilon
$$

with weights matrix (1.2) a Group Interaction model. If $\beta=0$ in equation (1.3) we say that the model is pure. Note that the number of groups is $r:=\sum_{i=1}^{p} r_{i}$, and the sample size is $n=\sum_{i=1}^{p} r_{i} m_{i}$. We assume throughout that $m_{i} \geq 2$ for all $i$. If the group sizes are all equal (i.e., $p=1$ ) the Group Interaction model is said to be balanced, otherwise, when $p>1$, it is said to be unbalanced. In the balanced case $W$ consists of $r$ copies of $B_{m}$, so, letting $m$ be the common group size,

$$
W=I_{r} \otimes B_{m}
$$

The class of Group Interaction models is discussed briefly in Hillier and Martellosio (2016) (hereafter H\&M), where some exact results are given for the pure balanced case. The main results from that paper are summarized in the next section. Then, in Section 3 we provide a complete analysis of the properties of $\hat{\lambda}_{\mathrm{ML}}$, and of exact inference procedures based upon it, for the pure balanced model. Results for the balanced model are of interest for their own sake, but also because this model is often used to illustrate theoretical results in the literature (see Lee (2004), (2007), and Lee, Liu, and Lin (2010), for instance). However, the balanced model is certainly of limited practical importance, so in Section 4 we go on to discuss the unbalanced model. For reasons to be explained, results for this model are much more complex than those for the balanced model. Thus, although we do give some general results, we often confine ourselves to the case of just two group sizes $(p=2)$ for simplicity. Proofs of the results that are not established directly in the main text, derivations, and some additional discussion associated with this article are provided online in supplementary material, available at Cambridge Journals Online (journals.cambridge.org/ect). The supplement also contains some additional graphics illustrating results mentioned in the article. 


\section{Preliminaries}

For the present, let $W$ be any $n \times n$ matrix with at least one negative, and at least one positive, eigenvalue, and normalized to have largest eigenvalue unity. The parameter space for $\lambda$ is taken to be the largest interval containing the origin within which the matrix $S_{\lambda}:=I_{n}-\lambda W$ remains non-singular. Letting $\omega_{\text {min }}$ denote the smallest real eigenvalue of $W$, the parameter space will thus be

$$
\Lambda:=\left(\omega_{\min }^{-1}, 1\right)
$$

We assume that the parameters are estimated by (quasi-) maximum likelihood (QML), where the likelihood adopted is that which would apply if, in equation (1.3), $\varepsilon \sim \mathrm{N}\left(0, I_{n}\right)$. We define the QMLE of $\lambda$ (assuming it exists), $\hat{\lambda}_{\mathrm{ML}}$, by

$$
\hat{\lambda}_{\mathrm{ML}}:=\arg \max _{\lambda \in \Lambda} l(\lambda),
$$

where $l(\lambda)$ is the profile (quasi) log-likelihood for $\lambda$, after maximization with respect to $\left(\beta, \sigma^{2}\right)$. This estimator is, in general, a zero of a high degree polynomial in $\lambda$, and thus cannot be written in closed form. However, it is shown in H\&M that, if $W$ has only real eigenvalues - which will be the case in the present paper - the profile likelihood $l(\lambda)$ is single-peaked on $\Lambda$. This means that, for each $z \in \Lambda$, the event that $\hat{\lambda}_{\mathrm{ML}} \leq z$ is identical to the event that the profile score at $z$, which we denote by $s(z)$, is negative. Thus, notwithstanding its unavailability in closed form, an exact expression for the distribution function (cdf) of $\hat{\lambda}_{\mathrm{ML}}$ can be written down immediately:

$$
\operatorname{Pr}\left(\hat{\lambda}_{\mathrm{ML}} \leq z ; \lambda\right)=\operatorname{Pr}(s(z) \leq 0)
$$

where, here and throughout, $\operatorname{Pr}\left(\hat{\lambda}_{\mathrm{ML}} \leq z ; \lambda\right)$ denotes the cdf of $\hat{\lambda}_{\mathrm{ML}}$ at the point $z \in \Lambda$ when the true parameter value is $\lambda \in \Lambda$. This representation is the basis for all of the results in this paper.

In addition to this single-peaked property, it is also easy to see that $s(z) \rightarrow-\infty$ as $z \rightarrow 1$ (from the left), and $s(z) \rightarrow+\infty$ as $z \rightarrow \omega_{\text {min }}^{-1}$ (from the right). Thus, $\operatorname{Pr}\left(\hat{\lambda}_{\mathrm{ML}} \leq\right.$ $z ; \lambda)=\operatorname{Pr}(s(z) \leq 0) \rightarrow 1$ as $z \rightarrow 1$, and $\operatorname{Pr}(s(z) \leq 0) \rightarrow 0$ as $z \rightarrow \omega_{\text {min }}^{-1}$. In other words, the inequality $\operatorname{Pr}(s(z) \leq 0)$ does indeed define a distribution function supported on $\Lambda$, as one would expect. Note that this argument holds whatever the distribution of $y$, provided only that the distribution of the random variable $s(z)$ is supported on the entire interval $\Lambda$. 
In the analytical results to follow we take the distribution of $\varepsilon$ to be $\mathrm{N}\left(0, I_{n}\right)$ (that is, the likelihood is correctly specified), but, as discussed in $\mathrm{H} \& \mathrm{M}$, all results obtained under this assumption continue to hold under scale mixtures of the $\mathrm{N}\left(0, I_{n}\right)$ distribution, the family we denote by $\operatorname{SMN}\left(0, I_{n}\right)$. For symmetric pure SAR models, equation (2.1) provides the following representation of the cdf of the MLE: ${ }^{3}$

$$
\operatorname{Pr}\left(\hat{\lambda}_{\mathrm{ML}} \leq z ; \lambda\right)=\operatorname{Pr}\left(\sum_{i=1}^{N} d_{i i}(z, \lambda) \chi_{n_{i}}^{2} \leq 0\right),
$$

where $N$ denotes the number of distinct eigenvalues of $W$ (which we take to be labelled in increasing order of magnitude), $n_{i}$ denotes the algebraic multiplicity of the eigenvalue $\omega_{i}$ of $W$, the $\chi_{n_{i}}^{2}$ variates are independent, and the coefficient functions $d_{i i}(z, \lambda)$ are given by

$$
d_{i i}(z, \lambda):=2\left(\frac{1-z \omega_{i}}{1-\lambda \omega_{i}}\right)^{2}\left(g_{i}(z)-\bar{g}(z)\right)
$$

In this expression, the

$$
g_{i}(z):=\frac{\omega_{i}}{1-z \omega_{i}},
$$

for $i=1, \ldots, N$, are the distinct eigenvalues of $G_{z}:=W S_{z}^{-1}$, where $S_{z}:=I_{n}-z W$, while $\bar{g}(z):=(1 / n) \sum_{i=1}^{N} n_{i} g_{i}(z)=(1 / n) \operatorname{tr}\left(G_{z}\right)$ is the average of the eigenvalues of $G_{z}$. Notice particularly that the distribution defined by (2.2) depends only on $\lambda$, and not on the nuisance parameter $\sigma^{2}$. In what follows we use the notation that, for any matrix $A$ of full column rank, $P_{A}:=A\left(A^{\prime} A\right)^{-1} A^{\prime}$, and $M_{A}:=I-P_{A}$. Also, $\operatorname{col}(A)$ denotes the column space of a matrix $A$. All matrices are assumed to be real.

\section{The Balanced Model}

In this section we first of all provide a complete analysis of the exact properties of $\hat{\lambda}_{\mathrm{ML}}$, and inference procedures based upon it, for the pure balanced model. Then, we consider some generalizations of these results to balanced models with regressors: we show that, for certain special choices of $X$, the results obtained for the pure model apply with only minor modifications. We note that in the pure balanced Group Interaction model, because the profile score is a quadratic in $\lambda, \hat{\lambda}_{\mathrm{ML}}$ is in fact available in closed form. However, its distribution theory is most easily obtained by using equation (2.2), and this also leads naturally to generalizations to the unbalanced model, when the estimator is typically not available in closed form.

\footnotetext{
${ }^{3}$ If normality is not assumed equation $(2.2)$ involves $T$ quadratic forms in $n_{i}$-dimensional vectors; see H\&M.
} 


\subsection{Distribution Function and Density}

For the pure balanced model we have $N=2, n_{1}=r(m-1), n_{2}=r, \Lambda=(-(m-1), 1)$, and the coefficients in equation (2.2) are given by

$$
\begin{aligned}
& d_{11}=-2\left(\frac{z+m-1}{\lambda+m-1}\right)^{2} \frac{1}{(\lambda+m-1)(1-\lambda)}<0, \\
& d_{22}=2\left(\frac{1-z}{1-\lambda}\right)^{2} \frac{(m-1)}{(\lambda+m-1)(1-\lambda)}>0 .
\end{aligned}
$$

Eliminating irrelevant scalars in (2.2), we obtain

$$
\operatorname{Pr}\left(\hat{\lambda}_{\mathrm{ML}} \leq z ; \lambda\right)=\operatorname{Pr}\left((m-1) \chi_{r}^{2} \leq c(z, \lambda) \chi_{r(m-1)}^{2}\right)
$$

where

$$
c(z, \lambda):=\left(\frac{(1-\lambda)(z+m-1)}{(1-z)(\lambda+m-1)}\right)^{2} .
$$

Thus, as stated in H\&M, in the pure balanced Group Interaction model with $\varepsilon \sim$ $\operatorname{SMN}\left(0, I_{n}\right)$, the cdf of $\hat{\lambda}_{\mathrm{ML}}$ is, for any $z, \lambda \in \Lambda$,

$$
\operatorname{Pr}\left(\hat{\lambda}_{\mathrm{ML}} \leq z ; \lambda\right)=\operatorname{Pr}\left(\mathrm{F}_{r, r(m-1)} \leq c(z, \lambda)\right)
$$

where $\mathrm{F}_{\nu_{1}, \nu_{2}}$ denotes a random variable distributed with $F$ distribution with $\nu_{1}$ and $\nu_{2}$ de-

grees of freedom. As expected, the distribution does not depend on $\sigma^{2}$. The corresponding density function is

$$
\operatorname{pdf}_{\hat{\lambda}_{\mathrm{ML}}}(z ; \lambda)=\frac{2 m \tau^{r(m-1)}}{B\left(\frac{r}{2}, \frac{r(m-1)}{2}\right)} \frac{(1-z)^{r(m-1)-1}(z+m-1)^{r-1}}{\left(\tau^{2}(1-z)^{2}+(z+m-1)^{2}\right)^{\frac{r m}{2}}},
$$

where $\tau:=\theta(\lambda) \sqrt{m-1}$, with

$$
\theta(\lambda)=\theta:=\frac{\lambda+m-1}{1-\lambda}>0 .
$$

The parameter $\theta$ is a 1-1 function of $\lambda$, and it is clear from equation (3.2) that the properties of $\hat{\lambda}_{\mathrm{ML}}$ depend on $\lambda$ only through $\theta$. This key parameter can be interpreted as just another way of locating the point $\lambda$ in the interval $\Lambda$, i.e., as a different parameterization of the model.

Note that $c(z, \lambda)=(\theta(z) / \theta(\lambda))^{2}$, and that $c(z, \lambda)$ is monotonic increasing in $z$. In fact, $c(z, \lambda) \rightarrow \infty$ as $z \rightarrow 1$, while $c(z, \lambda) \rightarrow 0$ as $z \rightarrow-(m-1)$. Hence, as noted in the comments following equation (2.1), equations (3.2) and (3.3) define a cdf and pdf supported on $\Lambda$. In addition, $\operatorname{Pr}\left(\hat{\lambda}_{\mathrm{ML}} \leq z ; \lambda\right) \rightarrow 0$ for all $z \in \Lambda$ as $\lambda \rightarrow 1$, because $c(z, \lambda) \rightarrow 0$, and 
$\operatorname{Pr}\left(\hat{\lambda}_{\mathrm{ML}} \leq z ; \lambda\right) \rightarrow 1$ for all $z \in \Lambda$ as $\lambda \rightarrow-(m-1)$, because $c(z, \lambda) \rightarrow \infty$. That is, the distribution of $\hat{\lambda}_{\mathrm{ML}}$ becomes degenerate, i.e., $\operatorname{var}\left(\hat{\lambda}_{\mathrm{ML}}\right) \rightarrow 0$, as $\lambda$ approaches either endpoint of $\Lambda$.

Finally, observe that, since $c(\lambda, \lambda)=1$, the probability that $\hat{\lambda}_{\mathrm{ML}}$ underestimates $\lambda$, $\operatorname{Pr}\left(\hat{\lambda}_{\mathrm{ML}} \leq \lambda ; \lambda\right)$, is given by $\operatorname{Pr}\left(\mathrm{F}_{r, r(m-1)} \leq 1\right)$, which does not depend on $\lambda$. The fact that $\operatorname{Pr}\left(\hat{\lambda}_{\mathrm{ML}} \leq \lambda ; \lambda\right)$ does not converge to 1 as $\lambda \rightarrow 1$, as might have been anticipated, is a consequence of the degeneracy of the distribution of $\hat{\lambda}_{\mathrm{ML}}$ just discussed.

Remark 3.1. Gaussian pure SAR models are members of the 2-parameter exponential family, with parameters $\left(\lambda, \sigma^{2}\right)$, the sufficient statistics being the three quadratic forms $q_{1}:=y^{\prime} y, q_{2}:=y^{\prime} W^{\prime} W y, q_{3}:=y^{\prime}\left(W+W^{\prime}\right) y$, and canonical parameters $\eta_{1}:=-1 /\left(2 \sigma^{2}\right)$, $\eta_{2}:=-\lambda^{2} /\left(2 \sigma^{2}\right), \eta_{3}:=\lambda /\left(2 \sigma^{2}\right)$. Thus, pure SAR models are, in the notation of BarndorffNielsen (1980), at worst, (3,2)-curved exponential models. In the balanced Group Interaction model with $W=I_{r} \otimes B_{m}$, these three sufficient statistics are not minimal, and can be written in terms of just two statistics,

$$
s_{1}:=y^{\prime}\left(I_{r} \otimes M_{\iota_{m}}\right) y, s_{2}:=y^{\prime}\left(I_{r} \otimes P_{\iota_{m}}\right) y
$$

Specifically, $q_{1}=s_{1}+s_{2}, q_{2}=s_{1} /(m-1)^{2}+s_{2}$, and $q_{3}=2\left(s_{2}-s_{1} /(m-1)\right)$. Collecting coefficients, the canonical parameters become

$$
\eta_{1}^{*}:=-\frac{1}{2 \sigma^{2}}\left(\frac{\lambda+m-1}{m-1}\right)^{2}, \eta_{2}^{*}:=-\frac{(1-\lambda)^{2}}{2 \sigma^{2}} .
$$

The pure balanced model is thus a regular exponential model, and it is this that makes it amenable to exact inference. We will see later that the unbalanced model cannot be reduced in this way, and so is genuinely curved. It can easily be checked that the two sufficient statistics $s_{1}$ and $s_{2}$ are independent in the balanced model, and

$$
\frac{s_{1}(\lambda+m-1)^{2}}{\sigma^{2}(m-1)^{2}} \sim \chi_{r(m-1)}^{2}, \frac{s_{2}(1-\lambda)^{2}}{\sigma^{2}} \sim \chi_{r}^{2} .
$$

Note that the parameter $\theta$ is closely related to the canonical parameters in the exponential family representation of the model, specifically, by $\theta^{2}=(m-1)^{2} \eta_{1}^{*} / \eta_{2}^{*}$.

\subsubsection{First Consequences}

The function $c(z, \lambda)$, defined on $\Lambda \times \Lambda$, is strictly decreasing in $\lambda$ and strictly increasing in $z$. The first fact means that the distribution functions for different values of $\lambda$ do not cross, so $\lambda_{1}<\lambda_{2}$ implies that the cdf for $\lambda=\lambda_{1}$ lies entirely above that for $\lambda=\lambda_{2}$. That is, in 
a pure balanced Group Interaction model with $\varepsilon \sim \operatorname{SMN}\left(0, I_{n}\right)$, the $\operatorname{cdf} \operatorname{Pr}\left(\hat{\lambda}_{\mathrm{ML}} \leq z ; \lambda\right)$ is decreasing in $\lambda$ over $\Lambda$, for any $z \in \Lambda$, or, in other words, $\hat{\lambda}_{\mathrm{ML}}$ is stochastically increasing in $\lambda$. Also, since the mean of $\hat{\lambda}_{\mathrm{ML}}$ is $-(m-1)$ plus the area above the cdf, it follows immediately that the mean of $\hat{\lambda}_{\mathrm{ML}}$ is a monotonic increasing function of $\lambda$.

The second property of the function $c(z, \lambda)$ implies that $\operatorname{Pr}\left(\hat{\lambda}_{\mathrm{ML}} \leq z ; \lambda\right)=\operatorname{Pr}\left(c\left(\hat{\lambda}_{\mathrm{ML}}, \lambda\right) \leq\right.$ $c(z, \lambda))=\operatorname{Pr}\left(\mathrm{F}_{r, r(m-1)} \leq c(z, \lambda)\right)$, or that $c\left(\hat{\lambda}_{\mathrm{ML}}, \lambda\right) \sim \mathrm{F}_{r, r(m-1)}$. Thus, the MLE of $\theta$,

$$
\hat{\theta}_{\mathrm{ML}}:=\frac{\hat{\lambda}_{\mathrm{ML}}+m-1}{1-\hat{\lambda}_{\mathrm{ML}}},
$$

is distributed as $\theta \sqrt{\mathrm{F}_{r, r(m-1)}}$, which we can state as follows.

Proposition 3.1. In the pure balanced Group Interaction model with $\varepsilon \sim \operatorname{SMN}\left(0, I_{n}\right)$,

$$
\hat{\theta}_{\mathrm{ML}}^{2} \sim \theta^{2} \mathrm{~F}_{r, r(m-1)} .
$$

The result in Proposition 3.1 is strikingly simple, and in the following sections we explore several of its consequences. It is worth pointing out that it provides a very efficient method of simulating any properties of $\hat{\lambda}_{\mathrm{ML}}$ (or functions of $\hat{\lambda}_{\mathrm{ML}}$ ) that are not available exactly, or are too complicated, by simply drawing samples from an $F$ distribution.

\subsubsection{Asymptotics Under Mixed-Normality}

In the case $r \rightarrow \infty$ with $m$ fixed (fixed-domain asymptotics), the asymptotic distribution of $\hat{\lambda}_{\mathrm{ML}}$ is covered by the results in Lee (2004): $\hat{\lambda}_{\mathrm{ML}}$ is consistent and asymptotically normal as $r \rightarrow \infty$ with large- $r$ variance (based on the information matrix, assuming normality) given by

$$
v_{\lambda}:=\frac{(1-\lambda)^{2}(\lambda+m-1)^{2}}{2 r m(m-1)} .
$$

Note that, as $\lambda$ goes to either extreme of $\Lambda$, this exhibits the same degeneracy as does the exact variance - see Section 3.1. Lee's paper does not fully study the asymptotic properties of $\hat{\lambda}_{\mathrm{ML}}$ when $r$ is fixed and $m \rightarrow \infty$ (infill asymptotics). Using Proposition 3.1 , both the large- $r$ and the large- $m$ asymptotics are easily deduced, under our present mixed-normal assumptions, from the following two representations of the $\mathrm{F}_{r, r(m-1)}$ random variable involved:

$$
\mathrm{F}_{r, r(m-1)}=\frac{(m-1)\left(\frac{1}{r} \sum_{i=1}^{r} \chi_{1}^{2}\right)}{\frac{1}{r} \sum_{i=1}^{r} \chi_{m-1}^{2}}=\frac{\chi_{r}^{2}}{\frac{1}{m-1} \sum_{i=1}^{m-1} \chi_{r}^{2}},
$$

where all $\chi^{2}$ variates are independent. From the first of these expressions, we see easily that $\mathrm{F}_{r, r(m-1)} \stackrel{p}{\longrightarrow} 1$ as $r \rightarrow \infty$ with $m$ fixed, which implies, by Proposition 3.1 , that 
$\hat{\theta}_{\mathrm{ML}} \stackrel{p}{\longrightarrow} \theta$, and hence that $\hat{\lambda}_{\mathrm{ML}} \stackrel{p}{\longrightarrow} \lambda$, a simple example of Lee's (2004) much more general results. Application of the delta method also produces, from the first of these expressions, the known asymptotic normality result under fixed-domain asymptotics. However, the second expression shows that, as $m \rightarrow \infty$ with $r$ fixed, $\mathrm{F}_{r, r(m-1)} \stackrel{d}{\longrightarrow} \chi_{r}^{2} / r$. Thus, in fact $\hat{\lambda}_{\mathrm{ML}}$ converges to a random variable under this regime, so is inconsistent under infill asymptotics. The limiting distribution function and density of $\hat{\lambda}_{\mathrm{ML}}$ as $m \rightarrow \infty$ follow by combining this result with Proposition 3.1, which immediately gives the following result (also given in $\mathrm{H} \& \mathrm{M})$.

Proposition 3.2. In a pure balanced Group Interaction model with $\varepsilon \sim \operatorname{SMN}\left(0, I_{n}\right)$, the limiting cdf of $\hat{\lambda}_{\mathrm{ML}}$ as $m \rightarrow \infty$ with $r$ fixed is,

$$
\lim _{m \rightarrow \infty} \operatorname{Pr}\left(\hat{\lambda}_{\mathrm{ML}} \leq z ; \lambda\right)=\operatorname{Pr}\left(\chi_{r}^{2} \leq r\left(\frac{1-\lambda}{1-z}\right)^{2}\right),-\infty<z<1,
$$

for any $\lambda, z \in \Lambda$, and the associated limiting density is

$$
\lim _{m \rightarrow \infty} \operatorname{pdf}_{\hat{\lambda}_{\mathrm{ML}}}(z ; \lambda)=\frac{r^{\frac{r}{2}}(1-\lambda)^{r}}{2^{\frac{r}{2}-1} \Gamma\left(\frac{r}{2}\right)(1-z)^{r+1}} e^{-\frac{r}{2}\left(\frac{1-\lambda}{1-z}\right)^{2}} .
$$

Figure 1 plots the exact density (3.3) and large- $r$ approximation when $r=m=10$, for $z \in(-1,1)$, and $\lambda=-0.5,0,0.5$. Here and elsewhere we focus on the interval $(-1,1)$ because it seems to be most relevant in applications. These plots and similar graphical evidence suggest the tentative conclusion that the density of $\hat{\lambda}_{\mathrm{ML}}$ is, in general, wellcentered on the true value of $\lambda$. The large- $r$ asymptotic approximation seems unsatisfactory even for this sample size, which is essentially what motivates an exact analysis based on the density (3.3).

In Figure 2 we also plot the exact density for $m=5,50$ and $r=10$, together with the large- $m$ approximation (3.6), which of course does not depend on $m$. Note that when $\lambda$ is positive the density of $\hat{\lambda}_{\mathrm{ML}}$ is quite insensitive to $m$, and the large- $m$ density gives an excellent approximation when $\lambda$ is positive (despite the MLE not converging in probability to a constant as $m \rightarrow \infty)$. This is due to the fact that in this model information about $\lambda$ grows very slowly with $m$. The approximation is less accurate when $\lambda$ is negative. 

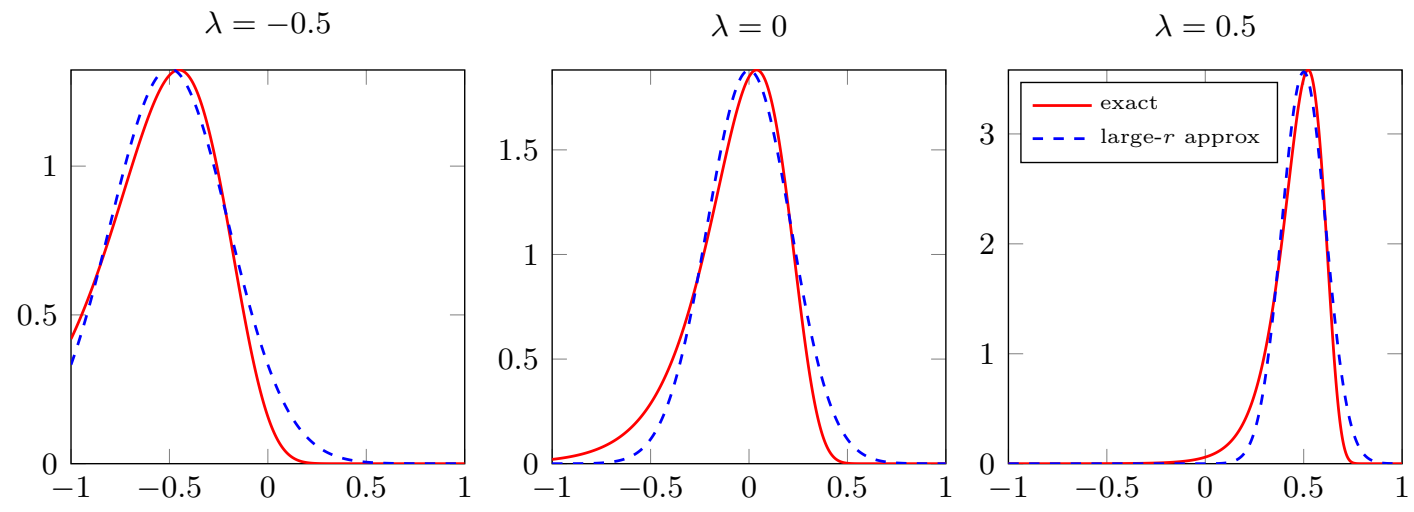

Figure 1: Density of $\hat{\lambda}_{\mathrm{ML}}$ for the pure balanced Group Interaction model with $\varepsilon \sim$ $\operatorname{SMN}\left(0, I_{n}\right)$, when $r=m=10$.
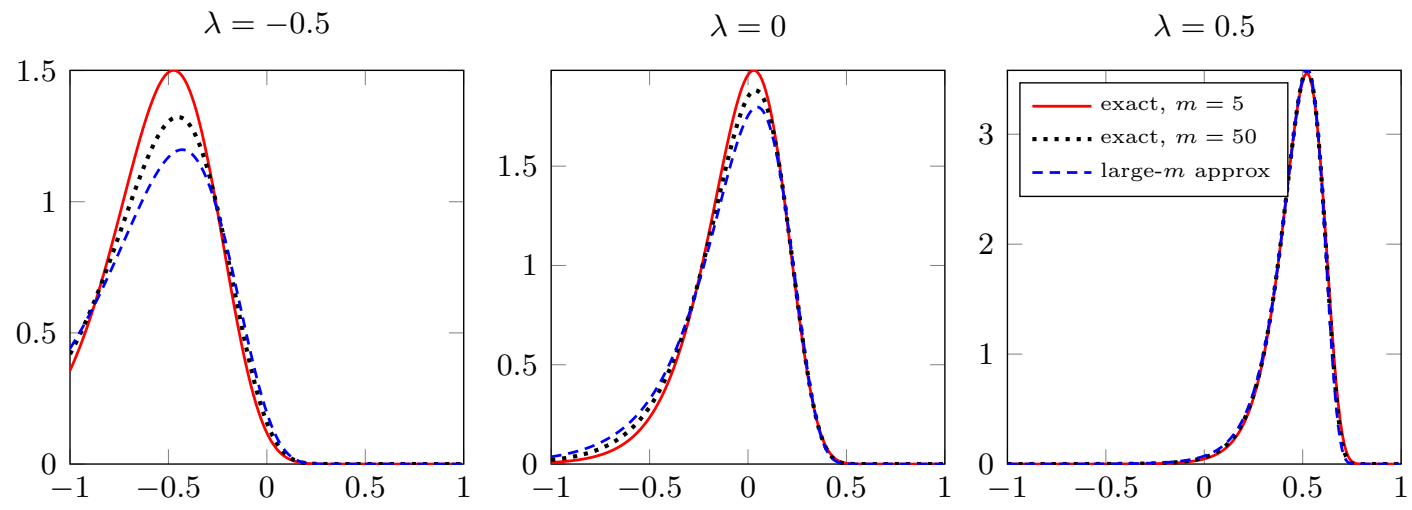

Figure 2: Density of $\hat{\lambda}_{\mathrm{ML}}$ for the pure balanced Group Interaction model with $\varepsilon \sim$ $\operatorname{SMN}\left(0, I_{n}\right)$, when $r=10$. 


\section{$3.2 \quad$ A Median Unbiased Estimator}

A second consequence of Proposition 3.1, along with the fact that $\theta$ is a monotonic function of $\lambda$, is that the median of $\hat{\lambda}_{\mathrm{ML}}$ is defined, in an obvious notation, by the identity $\operatorname{med}\left(\hat{\theta}_{\mathrm{ML}}\right)=\theta \sqrt{\operatorname{med}\left(\mathrm{F}_{r, r(m-1)}\right)}$. Solving this equation yields:

Proposition 3.3. In the pure balanced Group Interaction model with $\varepsilon \sim \operatorname{SMN}\left(0, I_{n}\right)$, the median of $\hat{\lambda}_{\mathrm{ML}}$ is

$$
\operatorname{med}\left(\hat{\lambda}_{\mathrm{ML}}\right)=1-\frac{m}{1+\theta \sqrt{\operatorname{med}\left(\mathrm{F}_{r, r(m-1)}\right)}} .
$$

Thus, the median of $\hat{\lambda}_{\mathrm{ML}}$ is a simple function of the median of an $F$ distribution. The median bias of $\hat{\lambda}_{\mathrm{ML}}$ is then, for any $\lambda \in \Lambda$,

$$
b_{\text {med }}(\lambda):=\operatorname{med}\left(\hat{\lambda}_{\mathrm{ML}}\right)-\lambda=\frac{m}{1+\theta}-\frac{m}{1+\theta \sqrt{\operatorname{med}\left(\mathrm{F}_{r, r(m-1)}\right)}} .
$$

Recalling that $\mathrm{F}_{r, r(m-1)} \stackrel{d}{\longrightarrow} \chi_{r}^{2} / r$ as $m \rightarrow \infty$, we immediately obtain the large- $m$ median bias (the large- $r$ median bias is obviously zero),

$$
\lim _{m \rightarrow \infty} b_{\text {med }}(\lambda)=(1-\lambda)\left(1-\sqrt{\frac{r}{\operatorname{med}\left(\chi_{r}^{2}\right)}}\right) .
$$

The detailed properties of the median bias are discussed further in Appendix A.1 of the Supplement. The median bias of $\hat{\lambda}_{\mathrm{ML}}$ can be important in some circumstances, but fortunately it can be eliminated completely by exploiting the fact that $\operatorname{med}\left(\hat{\lambda}_{\mathrm{ML}}\right)$ is known to be a monotonically increasing function of $\lambda$. In fact, recalling that $\hat{\theta}_{\mathrm{ML}} \cong \theta \sqrt{\mathrm{F}_{r, r(m-1)}}$, we have that $\operatorname{med}\left(\hat{\theta}_{\mathrm{ML}} / \sqrt{\operatorname{med}\left(\mathrm{F}_{r, r(m-1)}\right)}\right)=\theta$, i.e., the corrected estimator

$$
\tilde{\theta}_{\mathrm{ML}}:=\hat{\theta}_{\mathrm{ML}} / \sqrt{\operatorname{med}\left(\mathrm{F}_{r, r(m-1)}\right)}
$$

is exactly median-unbiased for $\theta$. Since $\theta$ is a monotonically increasing function of $\lambda$, we can assert the following:

Proposition 3.4. In the pure balanced Group Interaction model with $\varepsilon \sim \operatorname{SMN}\left(0, I_{n}\right)$, the estimator

$$
\hat{\lambda}_{\text {med }}:=\frac{\tilde{\theta}_{\mathrm{ML}}-m+1}{1+\tilde{\theta}_{\mathrm{ML}}}
$$

is exactly median-unbiased for $\lambda$.

Andrews (1993) used a closely related argument for the AR(1) model to obtain a median unbiased estimator, a more difficult case. Here we have the advantage that the median function is known exactly, and is known to be strictly monotonic. 


\subsection{Exact Confidence Interval for $\lambda$}

Proposition 3.1 also allows the construction of exact confidence sets for $\lambda$. Denoting the $\alpha$-quantile of the $F$ distribution with $\left(v_{1}, v_{2}\right)$ degrees of freedom by $\mathrm{F}_{v_{1}, v_{2} ; \alpha}$, we have ${ }^{4}$

$$
\operatorname{Pr}\left(\frac{\hat{\theta}_{\mathrm{ML}}}{\sqrt{\mathrm{F}_{r, r(m-1), 1-\alpha / 2}}}<\theta<\frac{\hat{\theta}_{\mathrm{ML}}}{\sqrt{\mathrm{F}_{r, r(m-1), \alpha / 2}}}\right)=1-\alpha .
$$

Turning this into a confidence interval for $\lambda$, we obtain:

Proposition 3.5. In the pure balanced Group Interaction model with $\varepsilon \sim \operatorname{SMN}\left(0, I_{n}\right)$, a $100(1-\alpha) \%$ exact confidence interval for $\lambda$ is

$$
\left(-(m-1)+\frac{m \hat{\theta}_{\mathrm{ML}}}{\hat{\theta}_{\mathrm{ML}}+\sqrt{\mathrm{F}_{r, r(m-1), 1-\alpha / 2}}}, 1-\frac{m \sqrt{\mathrm{F}_{r, r(m-1), \alpha / 2}}}{\hat{\theta}_{\mathrm{ML}}+\sqrt{\mathrm{F}_{r, r(m-1), \alpha / 2}}}\right) .
$$

In Appendix A.2 of the Supplement we discuss the properties of these intervals, and compare them with the commonly used large- $r$ confidence intervals.

\subsection{Exact Moments}

We first discuss the moments of the MLE for $\theta, \hat{\theta}_{\mathrm{ML}}$, and, since $\lambda$ is likely to remain the main parameter of interest, then go on to discuss the moments of $\hat{\lambda}_{\mathrm{ML}}$ itself. From Proposition 3.1 it is easily seen that $\hat{\theta}_{\mathrm{ML}}$ has moments (subject to existence) given by

$$
\mathrm{E}\left(\hat{\theta}_{\mathrm{ML}}^{s}\right)=\tau^{s} \mathrm{E}\left(f_{r, r(m-1)}^{\frac{s}{2}}\right),
$$

where $f_{v_{1}, v_{2}}$ denotes the ratio $\chi_{v_{1}}^{2} / \chi_{v_{2}}^{2}$ of two independent chi-square random variables. Since the density of $f_{r, r(m-1)}$ is

$$
\operatorname{pdf}_{f_{r, r(m-1)}}(f)=\frac{f^{\frac{r}{2}-1}(1+f)^{-\frac{r m}{2}}}{B\left(\frac{r}{2}, \frac{r(m-1)}{2}\right)},
$$

the integral defining the moments $\mathrm{E}\left(\hat{\theta}_{\mathrm{ML}}^{s}\right)$ exists for $s<r(m-1)$, and is of a standard form..$^{5}$

Proposition 3.6. In the pure balanced Group Interaction model with $\varepsilon \sim \operatorname{SMN}\left(0, I_{n}\right)$, the $s$-th moment of $\hat{\theta}_{\mathrm{ML}}$ exists only for $s<r(m-1)$, and in that case is given by

$$
\mathrm{E}\left(\hat{\theta}_{\mathrm{ML}}^{s}\right)=k_{s}(r, m) \tau^{s}, s<r(m-1),
$$

\footnotetext{
${ }^{4}$ As usual, there are many choices for such an interval at a given confidence level. Here we give an interval with equal tail areas, which is not necessarily the shortest, of course.

${ }^{5}$ The integral is of the type $\int_{0}^{\infty} f^{\alpha-1}(1+f)^{-(\alpha+\beta)} d f=\Gamma(\alpha) \Gamma(\beta) / \Gamma(\alpha+\beta)$, for $\alpha, \beta>0$.
} 
with

$$
k_{s}(r, m)=k_{s}:=\frac{\Gamma\left(\frac{r+s}{2}\right) \Gamma\left(\frac{r(m-1)-s}{2}\right)}{\Gamma\left(\frac{r}{2}\right) \Gamma\left(\frac{r(m-1)}{2}\right)} .
$$

Thus, although $\hat{\theta}_{\mathrm{ML}}$ itself is biased, the bias, because it is linear in $\theta$, is easily removed, and the variance of the corrected estimator easily computed. Turning to $\hat{\lambda}_{\text {ML }}$ itself, since the sample space for $\hat{\lambda}_{\mathrm{ML}}$ is bounded (and the density is bounded), it is clear that the moments of all orders of $\hat{\lambda}_{\mathrm{ML}}$ exist. However, it is difficult to express the integral defining the moments in terms of the density (3.3) in a useful closed form.

In Appendix A.3 of the Supplement we give some approximations to the moments derived from Proposition 3.6, and show that these can be used to construct an approximately unbiased estimator. There, we also compare i) the estimator $\hat{\lambda}_{\text {ML }}$ itself, ii) the approximately bias-corrected estimator, iii) the median-unbiased estimator introduced above, and iv) an indirect estimator also derived from Proposition 3.6, in terms of bias, median bias, and mean-square-error. Bao (2013) has recently obtained expansions for the mean of $\hat{\lambda}_{\mathrm{ML}}$ in the more general version of the model, equation (1.3) above, and used them to approximately bias-correct the estimator.

To conclude this section, observe that the large- $m$ asymptotic moments of $\hat{\lambda}_{\mathrm{ML}}$ can be obtained easily from the asymptotic density, equation (3.6), and are given, for $s<r$, by

$$
\lim _{m \rightarrow \infty} \mathrm{E}\left(\hat{\lambda}_{\mathrm{ML}}^{s}\right)=\sum_{j=0}^{s}\left(\begin{array}{l}
s \\
j
\end{array}\right) h_{r j}(\lambda-1)^{j},
$$

where

$$
h_{r j}:=\left(\frac{r}{2}\right)^{\frac{j}{2}} \frac{\Gamma\left(\frac{r-j}{2}\right)}{\Gamma\left(\frac{r}{2}\right)},
$$

with $h_{r 0}:=1$. Thus, the large- $m$ distribution has mean

$$
\lim _{m \rightarrow \infty} \mathrm{E}\left(\hat{\lambda}_{\mathrm{ML}}\right)=1+h_{r 1}(\lambda-1)
$$

and variance

$$
\lim _{m \rightarrow \infty} \operatorname{var}\left(\hat{\lambda}_{\mathrm{ML}}\right)=\left(h_{r 2}-h_{r 1}^{2}\right)(1-\lambda)^{2} .
$$

The limiting bias for large $m$ is thus $\lim _{m \rightarrow \infty} \mathrm{E}\left(\hat{\lambda}_{\mathrm{ML}}-\lambda\right)=(1-\lambda)\left(1-h_{r 1}\right)$, which is negative for all $r$ and $\lambda$, but diminishes rapidly as $r$ increases. Of course, the large- $r$ asymptotic mean $(\lambda)$ and variance $\left(v_{\lambda}\right)$ are known too. The limiting variances under the two asymptotic regimes can be very different, and we show in the Supplement that neither approximates the exact variance very well. 


\subsection{Hypothesis Testing: Best Invariant Test}

As we have seen, the pure balanced model is a two-parameter regular exponential model. In the canonical parameterization of Remark 3.1 the two sufficient statistics are $s_{1}:=$ $y^{\prime}\left(I_{r} \otimes M_{\iota_{m}}\right) y$, and $s_{2}:=y^{\prime}\left(I_{r} \otimes P_{\iota_{m}}\right) y$, with the distribution properties stated in Remark 3.1. The problem of testing $H_{0}: \lambda=0$ is invariant under the group of scale changes $s_{1} \rightarrow a s_{1}, s_{2} \rightarrow a s_{2}, a>0$, applied to the sufficient statistics, and under this group the statistic $s_{2} / s_{1}$ is a (single) maximal invariant. The MLE $\hat{\lambda}_{\mathrm{ML}}$ is itself invariant, therefore also maximal, since both are one-dimensional. The class of invariant tests in this model therefore coincides with the class of tests based on $\hat{\lambda}_{\mathrm{ML}} \cdot{ }^{6}$ Since we know the distribution of $\hat{\lambda}_{\mathrm{ML}}$ (under the $\operatorname{SMN}\left(0, I_{n}\right)$ assumption), we can apply the Neyman Pearson Lemma to the distribution of $\hat{\lambda}_{\mathrm{ML}}$ to obtain the uniformly most powerful invariant (UMPI) test of $H_{0}$ against each one-sided alternative. The resulting test can be shown to coincide with the Moran test (see King (1981), who gives an analogous result for the case $r=1$ ). ${ }^{7}$ Recently, Robinson and Rossi (2015b) have derived Edgeworth size-corrections for the OLS-based test statistic for this hypothesis in a general pure model. In our special case the test is exact.

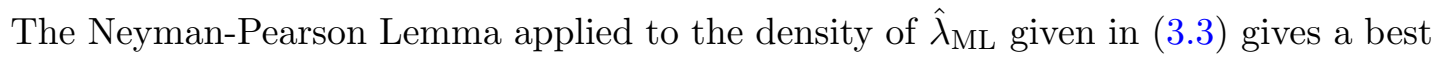
critical region consisting of large values of the likelihood ratio

$$
\frac{\operatorname{pdf}_{\hat{\lambda}_{\mathrm{ML}}}(z ; \lambda)}{\operatorname{pdf}_{\hat{\lambda}_{\mathrm{ML}}}(z ; 0)} \propto\left(\frac{1+\frac{1}{m-1} U(z)}{1+\frac{(m-1)}{\theta^{2}} U(z)}\right)^{\frac{r m}{2}}
$$

where $U(z):=((z+m-1) /((m-1)(1-z)))^{2}$. This ratio is increasing or decreasing in $U(z)$ as $\theta /(m-1) \gtrless 1$, so the best invariant test rejects $H_{0}$ against alternatives $\lambda>0$ when $U\left(\hat{\lambda}_{\mathrm{ML}}\right)=\left(\hat{\theta}_{\mathrm{ML}} /(m-1)\right)^{2}$ is large, and rejects against alternatives $\lambda<0$ when $U\left(\hat{\lambda}_{\mathrm{ML}}\right)$ is small. The critical values for a two-sided test can be derived directly from the $F_{r, r(m-1)}$ distribution, since, under $H_{0}, U\left(\hat{\lambda}_{\mathrm{ML}}\right) \sim F_{r, r(m-1)}$. Noting that, in the canonical representation of the model, $(m-1) s_{2} / s_{1}$ is the MLE for the parameter $(\theta /(m-1))^{2}$, we can therefore state:

Proposition 3.7. In the pure balanced Group Interaction model with $\varepsilon \sim \operatorname{SMN}\left(0, I_{n}\right)$, the UMPI test of $H_{0}: \lambda=0$ against alternatives $H_{1}^{+}: \lambda>0\left(H_{1}^{-}: \lambda<0\right)$ rejects $H_{0}$ when

\footnotetext{
${ }^{6}$ The likelihood ratio test is also invariant, therefore also based on $s_{1} / s_{2}$, or $\hat{\lambda}_{\mathrm{ML}}$, as can be shown directly. The same applies to a test based on a Studentized version of $\hat{\lambda}_{\mathrm{ML}}$, using, say, the estimated asymptotic variance as $r \rightarrow \infty$.

${ }^{7}$ As usual, of course, there is no uniformly best test against two-sided alternatives.
} 
$U\left(\hat{\lambda}_{\mathrm{ML}}\right)=(m-1) s_{2} / s_{1}$ is large (small). ${ }^{8}$ The test is exact, and critical values can be obtained from the fact that, under $H_{0}, U\left(\hat{\lambda}_{\mathrm{ML}}\right) \sim F_{r, r(m-1)}$.

When $H_{0}$ is false the test statistic $U\left(\hat{\lambda}_{\mathrm{ML}}\right)$ has the distribution

$$
U\left(\hat{\lambda}_{\mathrm{ML}}\right) \sim\left(\frac{\lambda+m-1}{(m-1)(1-\lambda)}\right)^{2} F_{r, r(m-1)},
$$

so that, for any critical value $t_{\alpha}$,

$$
\operatorname{Pr}\left(U\left(\hat{\lambda}_{\mathrm{ML}}\right)>t_{\alpha}\right)=\operatorname{Pr}\left(\mathrm{F}_{r, r(m-1)}>t_{\alpha}\left(\frac{(m-1)(1-\lambda)}{\lambda+m-1}\right)^{2}\right),
$$

with a similar expression for the other tail. For the one-sided test against $H_{1}^{+}: \lambda>0$, therefore, it is clear that the power $\rightarrow 1$ as $\lambda \rightarrow 1$, and the analogous conclusion holds as $\lambda \rightarrow-(m-1)$ for a one-sided test against $H_{1}^{-}: \lambda<0$. Exact power curves for the test(s) are easily obtained from equation $(3.13) .{ }^{9}$

\subsection{Balanced Models with Regressors}

The exact results derived above for the pure model do not generalize easily to the case of an arbitrary regressor matrix $X$. However, extensions are straightforward under certain specific assumptions on $X$, and we give some examples of this next. These examples are important in their own right, but also because they might suggest approximations for the case of an arbitrary $X$. Before continuing, we note that some care is required in dealing with the models with regressors, because there are choices for $X$ that mean that the number of sufficient statistics is less than the number of parameters, in which case inference (on the full parameter) is impossible. See Arnold (1979), Lee (2007), and H\&M for further discussion of this issue. The problem arises in the present balanced model when $\operatorname{col}(X)$ contains either of the two eigenspaces of $W$, which are $\operatorname{col}\left(I_{r} \otimes \iota_{m}\right)$ and $\operatorname{col}\left(I_{r} \otimes L_{m}\right)$, where $L_{m}$ is a matrix whose columns are a basis for the orthogonal complement of the span of $\iota_{m} \cdot{ }^{10}$ To rule this out we need the following assumption.

Assumption A. Neither $\operatorname{col}\left(I_{r} \otimes \iota_{m}\right)$ nor $\operatorname{col}\left(I_{r} \otimes L_{m}\right)$ is in $\operatorname{col}(X)$.

\footnotetext{
${ }^{8}$ The last equality here follows from the fact that, in the canonical representation of the model, $(m-$ 1) $s_{2} / s_{1}$ is the MLE for the parameter $(\theta /(m-1))^{2}$.

${ }^{9}$ An alternative approach would be to apply the Neyman-Pearson Lemma to the distribution of the statistic $s_{2} / s_{1}$ directly. It is straightforward but tedious to show that this yields exactly the same test as $\hat{\lambda}_{\mathrm{ML}}$ itself.

${ }^{10}$ If, for instance, $\operatorname{col}(X)$ contains $\operatorname{col}\left(I_{r} \otimes \iota_{m}\right)$, the term in the profile log-likelihood that involve $\lambda$ is $-n \log (\lambda+m-1)+\log \left(\left|S_{\lambda}\right|\right)$, so the profile score does not depend on the data.
} 
Note that $I_{r} \otimes \iota_{m}$ is the group fixed effect matrix. Hence, Assumption A requires, in particular, that the model does not contain group fixed effects. In model (1.3) with regressors and an arbitrary $W$, the random part of the log-likelihood is, under Gaussian assumptions,

$$
\left(S_{\lambda} y-X \beta\right)^{\prime}\left(S_{\lambda} y-X \beta\right)=(y-\lambda W y)^{\prime}(y-\lambda W y)+\beta^{\prime} X^{\prime} X \beta-2 \beta^{\prime} X^{\prime}(y-\lambda W y) .
$$

In general this cannot be written in terms of fewer than $2 k+3$ sufficient statistics, but in certain special cases reduction is possible. In the balanced Group Interaction model the first component can, as we have seen, be written in terms of $s_{1}, s_{2}$. The last term is in general a combination of both $X^{\prime} y$ and $X^{\prime} W y$, but it can be reduced to a single $k$-vector if $W^{\prime} X=X A$ for some $k \times k$ matrix $A$ (including $A=0$ ), that is, if $\operatorname{col}(X)$ is an invariant subspace of $W^{\prime}$. In this case the statistic $X^{\prime} y$ is sufficient. The case $A=0$ requires that the column space of $X$ is orthogonal to the column space of $W$, which, assuming $X$ is of full column rank $k$, can only be so if $\operatorname{rank}(W) \leq n-k$. This possibility therefore does not arise for the models studied in this paper, in which $W$ has full rank. But, for the balanced model, the column space of $X$ can indeed be an invariant subspace of $W^{\prime}$.

The simplest example of this is the case of a constant mean, i.e., $k=1$ and $X=\iota_{n}=$ $\iota_{r} \otimes \iota_{m}$. This model was considered in H\&M. More generally, we may have $X=\left(I_{r} \otimes \iota_{m}\right) R$, for some $r \times k$ matrix $R(k<r)$, in which case

$$
W^{\prime} X=\left(I_{r} \otimes B_{m}\right)\left(I_{r} \otimes \iota_{m}\right) R=\left(I_{r} \otimes B_{m} \iota_{m}\right) R=\left(I_{r} \otimes \iota_{m}\right) R=X .
$$

These cases entail that $\operatorname{col}(X)$ is spanned by eigenvectors of $W$ associated to the unit eigenvalue. Alternatively, $\operatorname{col}(X)$ may be spanned by eigenvectors associated to the eigenvalue $-1 /(m-1)$, or more generally, some combination of the two. If so we will have $X=\left(X_{1}, X_{2}\right)$, say, with $X_{1}$ of dimension $n \times k_{1}\left(k_{1}<r\right)$ and $X_{2}$ of dimension $n \times k_{2}$ $\left(k_{2}<r(m-1)\right)$, and with $\operatorname{col}\left(X_{1}\right) \subseteq \operatorname{col}\left(I_{r} \otimes \iota_{m}\right)$ and $\operatorname{col}\left(X_{2}\right) \subseteq \operatorname{col}\left(I_{r} \otimes L_{m}\right) .{ }^{11}$

In this circumstance the term $(y-\lambda W y)^{\prime} M_{X}(y-\lambda W y)$ that appears in the profile likelihood, and yields all of the results discussed earlier for the pure model, can instead be written as a linear combination of the two statistics

$$
\tilde{s}_{1}:=\tilde{y}_{1}^{\prime} M_{\tilde{X}_{1}} \tilde{y}_{1}, \tilde{s}_{2}:=\tilde{y}_{2}^{\prime} M_{\tilde{X}_{2}} \tilde{y}_{2},
$$

with the same coefficients as earlier. Here, $\tilde{y}:=H^{\prime} y$ and $\tilde{X}:=H^{\prime} X$, where $H:=\left(I_{r} \otimes\right.$ $\left.L_{m}, I_{r} \otimes l_{m}\right)$, with $l_{m}:=\iota_{m} / \sqrt{m}$, is an orthogonal matrix of eigenvectors of $W$. Thus,

\footnotetext{
${ }^{11}$ The inequalities $k_{1}<r$ and $k_{2}<r(m-1)$ must be strict for Assumption A to be satisfied.
} 
$\tilde{X}_{1}=\left(I_{r} \otimes L_{m}\right)^{\prime} X_{1}$ is $r(m-1) \times k_{1}$, and $\tilde{X}_{2}=\left(I_{r} \otimes l_{m}\right)^{\prime} X_{2}$ is $r \times k_{2}$. It is easily checked that

$$
\frac{\tilde{s}_{1}(\lambda+m-1)^{2}}{\sigma^{2}(m-1)^{2}} \sim \chi_{r(m-1)-k_{2}}^{2}, \frac{\tilde{s}_{2}(1-\lambda)^{2}}{\sigma^{2}} \sim \chi_{r-k_{1}}^{2} .
$$

It follows that the only changes needed to all of the above results, for models of this structure, are to the respective degrees of freedom of the F-variate involved in the expressions for the cdf. More precisely, we have established the following result, which generalizes formulae (3.2) and (3.3) to the case when $k_{1}$ and $k_{2}$ may be nonzero. ${ }^{12}$

Proposition 3.8. Suppose Assumption A holds and $\operatorname{col}(X) \subset \operatorname{col}\left(I_{r} \otimes \iota_{m}\right) \cup \operatorname{col}\left(I_{r} \otimes L_{m}\right)$. In the balanced Group Interaction model with $\varepsilon \sim \operatorname{SMN}\left(0, I_{n}\right)$, the cdf of $\hat{\lambda}_{\mathrm{ML}}$ is

$$
\operatorname{Pr}\left(\hat{\lambda}_{\mathrm{ML}} \leq z ; \lambda\right)=\operatorname{Pr}\left(\mathrm{F}_{v_{1}, v_{2}} \leq \frac{v_{2}}{v_{1}} \frac{c(z, \lambda)}{m-1}\right)
$$

for any $\lambda, z \in \Lambda$, where $v_{1}:=r-k_{1}$, with $k_{1}:=\operatorname{dim}\left(\operatorname{col}(X) \cap \operatorname{col}\left(I_{r} \otimes \iota_{m}\right)\right)<r$, and $v_{2}:=r(m-1)-k_{2}$, with $k_{2}:=k-k_{1}<r(m-1)$. The corresponding density is

$$
\operatorname{pdf}_{\hat{\lambda}_{\mathrm{ML}}}(z ; \lambda)=\frac{2 m \tau^{v_{2}}}{B\left(\frac{v_{1}}{2}, \frac{v_{2}}{2}\right)} \frac{(1-z)^{v_{2}-1}(z+m-1)^{v_{1}-1}}{\left(\tau^{2}(1-z)^{2}+(z+m-1)^{2}\right)^{\frac{n-k}{2}}} .
$$

It is certainly true that the conditions needed in Proposition 3.8 are restrictive, but they are met in some simple cases of practical interest, in addition to the constant mean case $X=\iota_{n}$. We briefly describe two of these in the next two subsections.

Remark 3.2. In the same setting as in Proposition $3.8\left(\operatorname{col}(X) \subset \operatorname{col}\left(I_{r} \otimes \iota_{m}\right) \cup \operatorname{col}\left(I_{r} \otimes\right.\right.$ $\left.L_{m}\right)$, but Assumption A holds), the Cliff-Ord test for $H_{0}: \lambda=0$ is UMPI against a one sided alternative in a mixed-Gaussian Group Interaction model. Here invariance is with respect to the group of transformations $y \rightarrow \kappa y+X \delta$ in the sample space, for any $\kappa>0$, any $\delta \in \mathbb{R}^{k}$; see King (1981). ${ }^{13}$

\footnotetext{
${ }^{12}$ Note that if $v_{1}=1$ the limit of the density (3.14) as $z \downarrow-(m-1)^{-1}$ is not zero. This can be seen as a consequence of the case $v_{1}=1$ being "close" to the degenerate case $v_{1}=0$, which causes $l(\lambda)$ to be unbounded from above in a neighborhood of $-(m-1)^{-1}$. We further note that Proposition 3.8 could also be derived directly from results in H\&M.

${ }^{13}$ If Assumption A does not hold, the Cliff-Ord statistic is degenerate, in the sense that it does not depend on the data, as the profile score is. As a consequence, the final paragraph of King (1981) needs to be interpreted with great care.
} 


\subsubsection{Individual Fixed Effects}

The model is

$$
y_{i}=\lambda B_{m} y_{i}+\mu+\sigma \varepsilon_{i}, i=1, . ., r
$$

where $y_{i} \in \mathbb{R}^{m}$ is the subvector of $y$ corresponding to $i$-th group, $\mu \in \mathbb{R}^{m}$ is a vector of individual fixed effects, so the groups have a common mean $\left(I_{m}-\lambda B_{m}\right)^{-1} \mu$, and a common autoregressive parameter $\lambda$. This is model (1.3) with $W=I_{r} \otimes B_{m}, X=\iota_{r} \otimes I_{m}$, and $\beta=\mu$. Proposition 3.8 applies with $k_{1}=1$ and $k_{2}=m-1$, and gives

$$
\operatorname{Pr}\left(\hat{\lambda}_{\mathrm{ML}} \leq z ; \lambda\right)=\operatorname{Pr}\left(\mathrm{F}_{r-1,(r-1)(m-1)} \leq c(z, \lambda)\right) .
$$

That is, as one might have expected, this case is analogous to a pure model having $r-1$ rather than $r$ copies of a complete graph on $m$ vertices. The asymptotics are thus the same as in Section 3.1.2: $\hat{\lambda}_{\mathrm{ML}}$ is consistent and asymptotically normal as $r \rightarrow \infty$, and converges in distribution to a random variable as $m \rightarrow \infty$ with $r$ fixed.

Remark 3.9. The model (3.15) is a special case of the spatial panel model studied in the recent paper by Robinson and Rossi (2015a), the difference being that in their paper $B_{m}$ in (3.15) is replaced by a general weights matrix $W$, common to the blocks, our $\mu$ is their $c$, and our $(r, m)$ are their $(T, n)$ (see also Lee and Yu (2010)). Under Robinson and Rossi's assumptions, $\hat{\lambda}_{\mathrm{ML}}$ is consistent and asymptotically normal as (their) $n$ goes to infinity, and they are able to obtain an Edgeworth expansion for the distribution of $\hat{\lambda}_{\mathrm{ML}}$. These results do not conflict with those just discussed, because, crucially, the matrix $B_{m}$ does not satisfy Assumption 3 (iv) in Robinson and Rossi (2015a).

\subsubsection{Group-Specific Regressions}

Consider now a balanced Group Interaction model with group specific $\beta$ coefficients:

$$
y_{i}=\lambda B_{m} y_{i}+X_{i} \beta_{i}+\sigma \varepsilon_{i}, i=1, . ., r,
$$

where the matrices $X_{i}$ are $m \times k_{i}$, with $k_{i} \leq m$, for all $i$. In this case $X=\bigoplus_{i=1}^{r} X_{i}(\bigoplus$ denoting matrix direct sum), $k=\sum_{i=1}^{r} k_{i}$, and $\beta^{\prime}=\left(\beta_{1}^{\prime}, . ., \beta_{r}^{\prime}\right)$ in equation (1.3). For each group one can check that the $k_{i}+3$ statistics $s_{1 i}=y_{i}^{\prime} M_{\iota_{m}} y_{i}, s_{2 i}=y_{i}^{\prime} P_{\iota_{m}} y_{i}, X_{i}^{\prime} y_{i}$, and $\iota_{m}^{\prime} y_{i}$ are sufficient for the $k_{i}+2$ parameters. The sums $s_{1}=\sum_{i=1}^{r} s_{1 i}$ and $s_{2}=\sum_{i=1}^{r} s_{2 i}$, together with the $X_{i}^{\prime} y_{i}, i=1, . ., r$, are therefore sufficient in the full model. If $\operatorname{col}\left(X_{i}\right)$ contains $\iota_{m}$ the statistic $\iota_{m}^{\prime} y_{i}$ is already accounted for in $X_{i}^{\prime} y_{i}$, so under this condition the model is regular for a single group. However, the condition $\iota_{m} \in \operatorname{col}\left(X_{i}\right)$ cannot be permitted for every $i$, for 
this would mean that $\operatorname{col}\left(I_{r} \otimes \iota_{m}\right)$ were a subspace of $\operatorname{col}(X)$, violating Assumption A. The alternative that also produces a regular model is that for those $i$ for which $\iota_{m} \notin \operatorname{col}\left(X_{i}\right)$, $\operatorname{col}\left(X_{i}\right) \subset \operatorname{col}\left(L_{m}\right)$. In this case the term involving $\iota_{m}^{\prime} y_{i}$ does not appear, and $X_{i}^{\prime} y_{i}$ is sufficient, again giving a regular model for that group. Note that $\operatorname{col}\left(X_{i}\right) \subset \operatorname{col}\left(L_{m}\right)$ would hold, for instance, if the elements of $X_{i}$ were deviations of the raw data from their respective within-group sample means. Assuming, therefore, that $\operatorname{col}\left(X_{i}\right) \subset \operatorname{col}\left(L_{m}\right)$ for $\rho \in(0, r)$ groups, and that, for the remaining $r-\rho$ groups, $\iota_{m} \in \operatorname{col}\left(X_{i}\right)$, the conditions of Proposition 3.8 are satisfied with $k_{1}=r-\rho$ and $k_{2}=k-r+\rho$, yielding the cdf

$$
\operatorname{Pr}\left(\hat{\lambda}_{\mathrm{ML}} \leq z ; \lambda\right)=\operatorname{Pr}\left(\mathrm{F}_{\rho, n-\rho-k} \leq \frac{n-\rho-k}{\rho(m-1)} c(z, \lambda)\right) .
$$

The asymptotics are then easily established. As $m \rightarrow \infty$ (keeping $r$ and $\rho$ fixed), $\hat{\lambda}_{\text {ML }}$ converges in distribution to a random variable (because $\mathrm{F}_{\rho, n-\rho-k} \stackrel{d}{\longrightarrow} \chi_{\rho}^{2} / \rho$ ). If $r \rightarrow \infty$ (keeping $m$ and $\rho$ fixed), the representation (3.18) implies that $\operatorname{Pr}\left(\hat{\lambda}_{\mathrm{ML}} \leq z ; \lambda\right) \rightarrow 1$, for any $\lambda, z \in \Lambda$, that is, $\hat{\lambda}_{\mathrm{ML}} \stackrel{p}{\longrightarrow}-(m-1)$. Next, writing $n-\rho-k=\rho(m-1)+(r-\rho) m-k$ shows that if $\rho \rightarrow \infty$ (keeping $r-\rho$ and $m$ fixed)

$$
\operatorname{Pr}\left(\hat{\lambda}_{\mathrm{ML}} \leq z ; \lambda\right)=I(1 \leq c(z, \lambda))=I\left(z \geq \frac{\theta-m+1}{1+\theta}\right)
$$

where $I(\cdot)$ is the indicator function, taking value 1 when its argument is true and 0 otherwise, and hence $\hat{\lambda}_{\mathrm{ML}} \stackrel{p}{\longrightarrow} \lambda$. One can also study intermediate cases. For instance, if both $r$ and $r-\rho$ diverge while $(n-\rho-k) /(\rho(m-1))$ remains bounded, a straightforward extension of the argument leading to equation (3.19) shows that $\hat{\lambda}_{\mathrm{ML}}$ converges in probability to an (in general) incorrect point in $\Lambda$.

We shall see below that in the unbalanced case there is no need to rule out the presence of group specific fixed effects. This will enable us to obtain a general exact representation of the cdf of $\hat{\lambda}_{\mathrm{ML}}$ in the case of group specific regressions.

\subsection{Conclusion on the Balanced Model}

The balanced Group Interaction model, the key property of which is that the spatial design matrix $W$ has just two distinct eigenvalues, is obviously a "toy" model, of the same status, perhaps, as the simple Gaussian regression model, and the AR(1) model in the time-series literature. Indeed, within the class of models in which $W$ is the adjacency matrix of a graph, it is the only model with just two distinct eigenvalues. Its practical relevance is obviously limited, but, as with the other examples mentioned, one hopes that study of its 
properties will be informative more generally. It goes without saying that one can only hope to obtain exact results under very restrictive assumptions, and we make no apology for beginning the study of exact inference in this class of models with its simplest member. However, in the interests of pragmatism, we now move on to the much more realistic, and therefore more complicated, unbalanced case.

\section{The Unbalanced Model}

The unbalanced Group Interaction model - with groups of different sizes - presents a much greater challenge, even for the pure model. In this section we present an exact result for the distribution of $\hat{\lambda}_{\mathrm{ML}}$, and some approximations to it. But, so far, we are unable to extend the detailed inference results obtained above for the balanced model to this more difficult case. The key difficulty is that some of the coefficients $d_{i i}(z, \lambda)$ in the expression for the cdf in equation (2.2) change sign as $z$ varies in $\Lambda$. This means that there are points in $\Lambda$ at which the cdf is non-analytic, and that the distribution has a different functional form in different sub-intervals of $\Lambda$. This makes analytical work with the exact distribution extremely difficult, if not impossible. Nevertheless, it is possible to make some progress by other means.

On the other hand, the presence of groups of different sizes has a favorable consequence: contrary to the balanced case, inference about $\lambda$ remains possible if (all) group specific fixed effects are included in the regression. We shall see that this immediately implies a simple representation of the cdf of $\hat{\lambda}_{\mathrm{ML}}$ that holds for general regressors, provided only that all $\beta$ parameters are group specific, and that group specific fixed effects are included.

In Sections 4.1-4.4 we restrict ourselves to the pure case, and often, for simplicity, we focus on the case of two group sizes (i.e., $p=2$ ). As is clear in equation (1.3), the interest-

parameter $\lambda$ is still assumed constant across groups. The case of group specific regressions is discussed briefly in Section 4.7 .

\subsection{Exact Representation}

In the unbalanced Group Interaction model each different group size introduces an extra distinct eigenvalue of $W$. If there are $r_{i}$ groups of distinct sizes $m_{i}, i=1, \ldots, p$, with $m_{1}$ the smallest group size, the eigenvalues of $W$ are: 1 , with multiplicity $r=\sum_{i=1}^{p} r_{i}$, and, 
for each $i=1, . ., p,-1 /\left(m_{i}-1\right)$ with multiplicity $n_{i}=r_{i}\left(m_{i}-1\right) .{ }^{14}$ The parameter space is therefore $\Lambda=\left(-\left(m_{1}-1\right), 1\right)$. The total sample size is $n=\sum_{i=1}^{p} r_{i} m_{i}$, and the number of distinct eigenvalues of $W$ is $N=p+1$. Since, for any Group Interaction model, $W$ is symmetric, the cdf of $\hat{\lambda}_{\mathrm{ML}}$ is, under mixed-Gaussian assumptions on $\varepsilon$, given by (2.2).

We will need the following property of the coefficient functions $g_{i}(z)-\bar{g}(z)$ in equation (2.3), proved in H\&M for any $W$ having only real eigenvalues: for any $z \in \Lambda$, the coefficients $g_{i}(z)-\bar{g}(z), i=1, . ., N$, are in increasing order (i.e., $j>i$ implies $g_{j}(z)>g_{i}(z)$ ). For any $z \in \Lambda, g_{1}(z)-\bar{g}(z)<0, g_{N}(z)-\bar{g}(z)>0$, and, for any $i=2, \ldots, N-1, g_{i}(z)-\bar{g}(z)$ changes sign exactly once on $\Lambda$.

We can divide the left-hand term in the inequality in (2.2) by the (positive) coefficient in the final term in the sum, giving the equivalent exact representation of the cdf,

$$
\operatorname{Pr}\left(\hat{\lambda}_{\mathrm{ML}} \leq z ; \lambda\right)=\operatorname{Pr}\left(\sum_{i=1}^{N} c_{i}(z, \lambda)\left(g_{i}(z)-\bar{g}(z)\right) \chi_{n_{i}}^{2} \leq 0\right),
$$

where, for the Group Interaction model considered here,

$$
c_{i}(z, \lambda):=\left(\frac{(1-\lambda)\left(z+m_{i}-1\right)}{(1-z)\left(\lambda+m_{i}-1\right)}\right)^{2}, i=1, . ., p,
$$

and $c_{N}(z, \lambda):=1$, all reducing to $c(z, \lambda)$ in equation (3.1) when the model is balanced. Since, for any $z \in \Lambda$, some of the $g_{i}(z)-\bar{g}(z)$ are positive and some are negative, it follows that $\sum_{i=1}^{N} c_{i}(z, \lambda)\left(g_{i}(z)-\bar{g}(z)\right) \chi_{n_{i}}^{2}$ reduces to the difference between two positive linear combinations of independent $\chi^{2}$ variates.

Remark 4.1. Notice that, for all $z \in \Lambda, c_{i}(z, \lambda) \rightarrow 0$ as $\lambda \rightarrow 1$, for each $i=1, . ., p$, while $c_{N}(z, \lambda)=1$. Since $g_{N}(z)-\bar{g}(z)>0$ for all $z \in \Lambda, \operatorname{Pr}\left(\hat{\lambda}_{\mathrm{ML}} \leq z ; \lambda\right) \rightarrow 0$ as $\lambda \rightarrow 1$. Likewise, as $\lambda \rightarrow-\left(m_{1}-1\right)$, all coefficients in (4.1), other than the first, remain finite, while $c_{1}(z, \lambda) \rightarrow \infty$ for all $z \in \Lambda$. Since $g_{1}(z)-\bar{g}(z)<0$ for all $z, \operatorname{Pr}\left(\hat{\lambda}_{\mathrm{ML}} \leq z ; \lambda\right) \rightarrow 1$ as $\lambda \rightarrow-\left(m_{1}-1\right)$. Thus, as in the balanced case, the distribution of $\hat{\lambda}_{\mathrm{ML}}$ becomes degenerate as $\lambda$ approaches the endpoints of $\Lambda$.

The eigenvalues of $G_{z}$ are $g_{i}(z)=-1 /\left(z+m_{i}-1\right), i=1, . ., p$, and $g_{p+1}(z)=1 /(1-z)$, so that

$$
\bar{g}(z)=\frac{z}{n(1-z)} \sum_{i=1}^{p} \frac{r_{i} m_{i}}{z+m_{i}-1},
$$

\footnotetext{
${ }^{14}$ The corresponding eigenspaces are $\operatorname{col}\left(\bigoplus_{i=1}^{p}\left(I_{r_{i}} \otimes \iota_{m_{i}}\right)\right)$ associated to the eigenvalue 1 and $\operatorname{col}\left(I_{r_{i}} \otimes\right.$ $\left.L_{m_{i}}\right)$ associated to $-1 /\left(m_{i}-1\right), i=1, \ldots, p$. It is easily verified that when $p=1$ the eigenstructure reduces to the one given in Section 3.
} 
and

$$
g_{i}(z)-\bar{g}(z)=-\frac{1}{n(1-z)} \sum_{j=1}^{p}\left(r_{j} m_{j}\left(\frac{1-z}{z+m_{i}-1}+\frac{z}{z+m_{j}-1}\right)\right),
$$

for $i=1, . ., p$, while

$$
g_{p+1}(z)-\bar{g}(z)=\frac{1}{n(1-z)} \sum_{i=1}^{p} \frac{r_{i} m_{i}\left(m_{i}-1\right)}{z+m_{i}-1} .
$$

Note that $\bar{g}(z)$ has the sign of $z$, and $\bar{g}(0)=0$. As noted earlier, $g_{1}(z)-\bar{g}(z)<0$ for all $z \in \Lambda, g_{p+1}(z)-\bar{g}(z)>0$ for all $z \in \Lambda$, and the remaining terms all change sign exactly once as $z$ traverses $\Lambda$. Thus, the number of positive and negative terms in the representation (4.1) varies with $z$. If the model is balanced $(p=1)$ the exact representation given here reduces to the result for the balanced case discussed earlier.

For any $p \geq 2$, let $z_{i}$ denote the unique point in $\Lambda$ at which $g_{i}(z)-\bar{g}(z)=0$, for each $i=2, \ldots, p$. The distribution of $\hat{\lambda}_{\mathrm{ML}}$ is non-analytic at the points $z_{i}$, and has a different functional form in each interval between successive points. The number of positive and negative terms in (4.1) remains the same within an interval, but the numbers of each differ in the different intervals.

Example 1 (Two Group Sizes). In the case $p=2$ we have, after simplification, ${ }^{15}$

$$
\begin{aligned}
g_{1}(z)-\bar{g}(z) & =-\frac{n\left(m_{2}-1\right)+z\left(n-r_{2} m_{2}\left(m_{2}-m_{1}\right)\right)}{n(1-z)\left(z+m_{1}-1\right)\left(z+m_{2}-1\right)}, \\
g_{2}(z)-\bar{g}(z) & =-\frac{n\left(m_{1}-1\right)+z\left(n+r_{1} m_{1}\left(m_{2}-m_{1}\right)\right)}{n(1-z)\left(z+m_{1}-1\right)\left(z+m_{2}-1\right)}, \\
g_{3}(z)-\bar{g}(z) & =\frac{r_{1} m_{1}\left(m_{1}-1\right)\left(z+m_{2}-1\right)+r_{2} m_{2}\left(m_{2}-1\right)\left(z+m_{1}-1\right)}{n(1-z)\left(z+m_{1}-1\right)\left(z+m_{2}-1\right)} .
\end{aligned}
$$

The first is always negative, the last always positive, for $z \in \Lambda$, while the second changes sign at

$$
z_{2}=-\frac{n\left(m_{1}-1\right)}{n+r_{1} m_{1}\left(m_{2}-m_{1}\right)}<0,
$$

being negative for $z>z_{2}$, positive for $z<z_{2}$.

After briefly discussing the asymptotic properties of $\hat{\lambda}_{\mathrm{ML}}$ that follow from the exact representation, we next discuss (again briefly) the distribution properties of linear combinations of independent $\chi^{2}$ variates with positive coefficients, a subject upon which there is a large literature.

\footnotetext{
${ }^{15}$ The common denominators of the coefficients here could obviously be dropped, but to economize on notation we do not do so.
} 


\subsection{Asymptotics in the Unbalanced Group Interaction Model}

The representation of the cdf of $\hat{\lambda}_{\mathrm{ML}}$ in equation (4.1) provides a useful starting point for deriving asymptotic properties of $\hat{\lambda}_{\mathrm{ML}}$ under the mixed Gaussian assumption. Different asymptotic regimes are possible, depending on how the $m_{i}$ 's and the $r_{i}$ 's are assumed to behave as the total sample size grows. To understand the issues we rewrite equation (4.1) in the form

$$
\operatorname{Pr}\left(\hat{\lambda}_{\mathrm{ML}} \leq z ; \lambda\right)=\operatorname{Pr}\left(\chi_{r}^{2}+\sum_{i=1}^{p} \psi_{i}(z, \lambda) \chi_{n_{i}}^{2} \leq 0\right)
$$

where

$$
\psi_{i}(z, \lambda):=c_{i}(z, \lambda) \frac{g_{i}(z)-\bar{g}(z)}{g_{p+1}(z)-\bar{g}(z)},
$$

for $i=1, \ldots, p$. Assuming $p$, the number of different group sizes, is fixed, one can consider two types of asymptotic regime. The first, infill asymptotics, holds the $r_{i}$ fixed (hence also $r$ ), and assumes one or more of the $m_{i}$ produce the increased sample size. The second, fixed-domain asymptotics, holds the $m_{i}$ fixed and assumes an increase in one or more of the $r_{i}$. This second case satisfies the assumptions in Lee (2004). Hence, it is already known that, under regularity conditions, $\hat{\lambda}_{\mathrm{ML}}$ is consistent and asymptotically normal, which is also easily deduced from the representation (4.4) by a characteristic function argument. In the first case Lee's (2004) results leave the properties of $\hat{\lambda}_{\mathrm{ML}}$ open. In fact, the situation is very much as in the balanced case: it is clear from (4.4) that, under infill asymptotics

in the unbalanced case, convergence will be to a random variable, because the term $\chi_{r}^{2}$ in (4.4) will be unaffected. Precise details for this situation depend on exactly what is assumed about the behaviour of the $m_{i}$, but $\hat{\lambda}_{\mathrm{ML}}$ is clearly again inconsistent under infill asymptotics.

\subsection{Exact distribution of a Positive Linear Combinations of $\chi^{2}$ Variates}

As we have just seen, we need to deal with pairs of statistics of the form

$$
Q_{s}:=\sum_{i=1}^{s} a_{i} \chi_{n_{i}}^{2}
$$

with all the $a_{i}$ positive. In our case these coefficients are functions of $z$.

Define the $n \times n$ diagonal matrix $\left(n=\sum_{i=1}^{s} n_{i}\right)$

$$
A=A_{n_{1}, \ldots, n_{s}}\left(a_{1}, \ldots, a_{s}\right):=\operatorname{diag}\left(a_{i} I_{n_{i}}, i=1, . ., s\right) .
$$

It is well known that the cumulants of $Q_{s}$ of all orders exist and are given by

$$
\kappa_{l}:=2^{l-1}(l-1) ! \operatorname{tr}\left(A^{l}\right)=2^{l-1}(j-1) ! \pi_{l},
$$


where $\pi_{l}:=\sum_{i=1}^{s} n_{i} a_{i}^{l}=\operatorname{tr}\left(A^{l}\right)$. These properties are quite simple, but, despite that, exact distribution theory for $Q_{s}$ is not straightforward, and there is a very large literature dealing with the subject. We briefly introduce some of this next. Let $\phi$ be a positive number such that $\phi a_{i} \geq 1$ for all $i$. An expression for the exact density is

$$
\operatorname{pdf}_{Q_{s}}(q)=\frac{|\phi A|^{-\frac{1}{2}}}{2^{\frac{n}{2}} \Gamma\left(\frac{n}{2}\right)} \exp \left(-\frac{1}{2} \phi q\right) q^{\frac{n}{2}-1}{ }_{1} F_{1}\left(\frac{1}{2}, \frac{n}{2} ; \frac{1}{2} q \phi\left(I_{n}-(\phi A)^{-1}\right)\right)
$$

(see James (1964), and Ruben (1962)). The hypergeometric function here is a confluent hypergeometric function with matrix argument (Muirhead (1982), Chapter 7), and this special function makes the distribution difficult. For $\phi$ such that $\phi a_{i}>1$ for all $i$, the distribution of $\phi Q_{s}$ can be expressed as a mixture of central $\chi^{2}$ distributions with weights

$$
p_{j}(\phi A):=\frac{\left(\frac{1}{2}\right)_{j}}{j !}|\phi A|^{-\frac{1}{2}} C_{j}\left(I_{n}-(\phi A)^{-1}\right),
$$

where $(a)_{j}:=a(a+1) \ldots(a+j-1)$ is the Pochhammer symbol, and $C_{j}(\cdot)$ denotes the top-order zonal polynomial of degree $j$ in the indicated matrix. It is easy to confirm that the $p_{j}(\phi A)$ are non-negative and sum to unity. The choice of $\phi>0$ is arbitrary subject to $\phi a_{i}>1$ for all $i$. The weights $p_{j}(\phi A)$ are relatively complicated polynomials in the $a_{i}$, and are difficult to interpret. ${ }^{16}$ See Ruben (1962) and Johnson, Kotz, and Balakrishnan (1994) for further details of these and related expansions. There is some incentive, therefore, to seek approximations to the distribution, and we discuss some of these briefly below.

In the case $s=2$, however, the result is reasonably simple. Without loss of generality we consider the distribution of a statistic of the form $a_{1} \chi_{v_{1}}^{2}+a_{2} \chi_{v_{2}}^{2}$, with $0<a_{1}<a_{2}$.

Proposition 4.1. Let $Q:=a_{1} \chi_{v_{1}}^{2}+a_{2} \chi_{v_{2}}^{2}$, with $0<a_{1}<a_{2}$. The density of $Q$ is given by

$$
\operatorname{pdf}_{Q}(q)=\frac{\phi^{\frac{v}{2}} \psi^{\frac{v_{2}}{2}} \exp \left(-\frac{\phi q}{2}\right) q^{\frac{v}{2}-1}}{2^{\frac{v}{2}} \Gamma\left(\frac{v}{2}\right)} F_{1}\left(\frac{v_{2}}{2}, \frac{v}{2} ; \frac{1}{2} \phi q(1-\psi)\right),
$$

where $\phi=1 / a_{1}, v:=v_{1}+v_{2}$, and $\psi:=a_{1} / a_{2}<1$.

The distribution function follows at once. Note that the confluent hypergeometric function in (4.9) has scalar argument, and is a built-in function in most modern mathematical packages. Equation (4.9) can be rewritten as

$$
\operatorname{pdf}_{Q}(q)=\phi \psi^{\frac{v_{2}}{2}} \sum_{k=0}^{\infty} \frac{\left(\frac{v_{2}}{2}\right)_{k}(1-\psi)^{k}}{k !} g_{v+2 k}(\phi q),
$$

\footnotetext{
${ }^{16}$ Recall that the non-central $\chi^{2}$ distribution also has this form, but with a Poisson mixing distribution with mean equal to the non-centrality parameter. This is obviously simpler than the present case.
} 
where $g_{\xi}(\cdot)$ denotes the density function of a $\chi_{\xi}^{2}$ random variable. That is, the distribution is a mixture of $\chi^{2}$ densities. This can be useful for some calculations, and for interpretation, but is perhaps less so for computation purposes.

\subsubsection{Approximations for Positive Definite Forms}

Because the exact distribution of a positive definite quadratic form is quite complicated, there is a clear incentive to approximate. And, because such forms are ubiquitous throughout statistics, there is a very large literature on the subject. The simplest approximation, usually attributed to Fisher, is to treat $Q_{s}$ as a multiple of a $\chi^{2}$ variate, $Q_{s}=\alpha \chi_{v}^{2}$, choosing $\alpha$ and $v$ so that the first two cumulants of the two distributions agree. This entails the choices $\alpha=\pi_{2} / \pi_{1}$ and $v=\pi_{1}^{2} / \pi_{2}$, where, as above, $\pi_{l}=\sum_{i=1}^{s} n_{i} a_{i}^{l}$.

A more sophisticated approximation due to Hall (1983) and Buckley and Eagleson (1988), is to use three parameters, with $Q_{s}=\alpha \chi_{v}^{2}+\beta$, and choosing $(\alpha, \beta, v)$ so that the first three cumulants agree. This entails the choices $\alpha=\pi_{3} / \pi_{2}, \beta=\pi_{1}-\pi_{2}^{2} / \pi_{3}$, and $v=\pi_{2}^{3} / \pi_{3}^{2}$. Buckley and Eagleson (1988) show that this representation can be formally justified by an argument based on Edgeworth expansions of the two distributions involved, and give explicit bounds on the error involved in approximating the distribution function in this way. Hall (1983) calls this a "penultimate" approximation to the distribution of $Q_{s}$, which of course, when suitably standardised, converges to a standard normal variate. For our purposes, the simpler two-cumulant approximation is more useful, and seems to work quite well. A number of other, typically more complicated, approximations are extant for a comprehensive discussion, see Johnson, Kotz, and Balakrishnan (1994).

Finally, one can use saddlepoint methods for inverting the characteristic function (Daniels (1987), Lugannani and Rice (1980)). We make use of this method in H\&M in the very general context of equation (1.3), and find that it works extremely well. ${ }^{17} \mathrm{We}$ do not discuss this further in the present paper.

\subsection{Exact Distribution of $\hat{\lambda}_{\mathrm{ML}}$}

From the exact results for a pair of independent positive linear combinations like those given above, one can easily obtain an exact formula for the probability $\operatorname{Pr}\left(Q_{1 t} \leq Q_{2 t}\right)$, with $Q_{i t}$ based on matrix $A_{i t}$, by simple transformation and integration. Thus, in each

\footnotetext{
${ }^{17}$ Peter Phillips, in a talk entitled "Some Magic with Saddlepoints", (Southampton, June 2014) derived the saddlepoint approximation for the OLS estimator of $\lambda$ in the balanced model. This is also extremely accurate.
} 
interval between points where one of the coefficients in (4.1) vanishes we have a different representation of the distribution - in fact a generalization of the result for the balanced model. The details are given in H\&M. However, this result is not particularly informative about the properties of the estimator. Instead, we seek accurate approximations to the exact results that are more easily interpreted, and more informative. Before considering that further, in the next section we give the exact results for the case of just two group sizes (i.e., $p=2$ ), which are reasonably tractable.

\subsubsection{Two Group Sizes (continued)}

When there are $p=2$ different group sizes the coefficients of the three $\chi^{2}$ variates in the sum in (4.1) have the following signs:

\begin{tabular}{cccc}
\hline & $\chi_{r_{1}\left(m_{1}-1\right)}^{2}$ & $\chi_{r_{2}\left(m_{2}-1\right)}^{2}$ & $\chi_{r}^{2}$ \\
\hline$z<z_{2}$ & - & + & + \\
$z>z_{2}$ & - & - & + \\
\hline
\end{tabular}

Using the coefficients in (4.5), we have, for $z<z_{2}$, where $\psi_{2}(z, \lambda)>0$,

$$
\operatorname{Pr}\left(\hat{\lambda}_{\mathrm{ML}} \leq z ; \lambda\right)=\operatorname{Pr}\left(\psi_{2}(z, \lambda) \chi_{n_{2}}^{2}+\chi_{r}^{2} \leq\left(-\psi_{1}(z, \lambda)\right) \chi_{n_{1}}^{2}\right)
$$

while for $z>z_{2}$, where $\psi_{2}(z, \lambda)<0$,

$$
\operatorname{Pr}\left(\hat{\lambda}_{\mathrm{ML}} \leq z ; \lambda\right)=\operatorname{Pr}\left(\chi_{r}^{2} \leq-\psi_{1}(z, \lambda) \chi_{n_{1}}^{2}+\psi_{2}(z, \lambda) \chi_{n_{2}}^{2}\right)
$$

Each of these involves a linear combination of two $\chi^{2}$ random variables with positive coefficients, and a third, independent $\chi^{2}$ variate. Expressions for the distribution functions in the two intervals can be obtained by applying the results in the previous subsection, but it is difficult to use those expressions to obtain information about the properties of $\hat{\lambda}_{\mathrm{ML}}$, in particular, its density. Here we pursue an alternative conditioning argument that is more successful.

Remark 4.2. Noting that $\psi_{2}\left(z_{2}, \lambda\right)=0$, and, as is easily verified, $-\psi_{1}\left(z_{2}, \lambda\right)=r / n_{1}$, we have, on setting $z=z_{2}$ in either of equations (4.10) or (4.11),

$$
\operatorname{Pr}\left(\hat{\lambda}_{\mathrm{ML}} \leq z_{2} ; \lambda\right)=\operatorname{Pr}\left(\mathrm{F}_{r, n_{1}}<c_{1}\left(z_{2}, \lambda\right)\right)
$$

For values of $r_{1}, r_{2}$ that are not too small this function (of $\lambda$ ) is near 1 for $\lambda<z_{2}$, and near zero for $\lambda>z_{2}$, falling sharply from 1 to 0 in the neighborhood of $z_{2}$. That is, for values 
$\lambda<z_{2} \hat{\lambda}_{\mathrm{ML}}$ is almost certainly below $z_{2}$, and for values $\lambda>z_{2}$ it is almost certainly above $z_{2}$. If $z_{2}<-1$, and $\lambda \in(-1,1)$, this implies that the distribution of $\hat{\lambda}_{\mathrm{ML}}$ will be almost entirely confined to the interval $z>z_{2}$. For $\lambda=z_{2}, \operatorname{Pr}\left(\hat{\lambda}_{\mathrm{ML}} \leq z_{2} ; z_{2}\right)=\operatorname{Pr}\left(\mathrm{F}_{r, n_{1}}<1\right)$, which is near .5 as long as $r / n_{1}$ is near 1 . Other evidence about the median will be discussed shortly.

Let $q_{v}$ denote a $\chi_{v}^{2}$ random variable. All such variables in the expressions to follow are independent. For $z<z_{2}$, we can condition on the variables $q_{r}$ and $q_{n_{2}}$ on the left in the expression for $\operatorname{Pr}\left(\hat{\lambda}_{\mathrm{ML}} \leq z ; \lambda\right)$, giving the conditional result

$$
\operatorname{Pr}\left(\hat{\lambda}_{\mathrm{ML}} \leq z \mid q_{r}, q_{n_{2}}, \lambda\right)=1-\mathcal{G}_{n_{1}}\left(\frac{q_{r}+\psi_{2}(z, \lambda) q_{n_{2}}}{-\psi_{1}(z, \lambda)}\right), \quad-\left(m_{1}-1\right)<z<z_{2},
$$

where $\mathcal{G}_{v}$ denotes the cdf of the $\chi_{v}^{2}$ random variable. For $z>z_{2}$, we can condition instead on $\left(q_{n_{1}}, q_{n_{2}}\right)$, giving

$$
\operatorname{Pr}\left(\hat{\lambda}_{\mathrm{ML}} \leq z \mid q_{n_{1}}, q_{n_{2}}, \lambda\right)=\mathcal{G}_{r}\left(-\left(\psi_{1}(z, \lambda) q_{n_{1}}+\psi_{2}(z, \lambda) q_{n_{2}}\right)\right), z_{2}<z<1,
$$

Expressions for the unconditional cdf's can be obtained from these by averaging, but we shall focus instead on the unconditional density in each interval. The reason that this is straightforward is that expressions for the conditional density are easily obtained from these conditional cdf's, and these can then be converted into the (components of the) unconditional density.

The expressions for the cdf here have a common form from which the density follows easily. The following result can be applied in both cases. ${ }^{18}$

Proposition 4.2. Let $a_{1}(z)$ and $a_{2}(z)$ be strictly positive functions of $z$ on some interval $\Lambda_{0}$. Let $q_{1} \sim \chi_{\alpha}^{2}, q_{2} \sim \chi_{\beta}^{2}$ be independent, and let $w$ be a random variable with conditional cdf, given $\left(q_{1}, q_{2}\right)$, given by

$$
\operatorname{Pr}\left(w \leq z \mid q_{1}, q_{2}\right)=\mathcal{G}_{\gamma}\left(a_{1} q_{1}+a_{2} q_{2}\right)
$$

for $z \in \Lambda_{0}$. The conditional density of $w$, given $\left(q_{1}, q_{2}\right)$, is given by

$$
\operatorname{pdf}_{w}\left(z \mid q_{1}, q_{2}\right)=\frac{\exp \left(-\frac{1}{2}\left(a_{1} q_{1}+a_{2} q_{2}\right)\right)}{2^{\frac{\gamma}{2}} \Gamma\left(\frac{\gamma}{2}\right)}\left(a_{1} q_{1}+a_{2} q_{2}\right)^{\frac{\gamma}{2}-1}\left(\dot{a}_{1} q_{1}+\dot{a}_{2} q_{2}\right),
$$

where the dot denotes the derivative with respect to $z$.

\footnotetext{
${ }^{18}$ The proof is omitted: the result is a simple application of Leibnitz's Theorem on the derivative of an integral (when the limits depend on the variable).
} 
Then, denoting the unconditional density of $w$ at $w=z$ when the parameters are $(\alpha, \beta, \gamma)$ by $\operatorname{pdf}_{w}(z ; \alpha, \beta, \gamma)$, we have (omitting the argument of the $a_{i}(\cdot)$ for simplicity):

Proposition 4.3. In the setting of Proposition 4.2, when $a_{1}>a_{2}$,

$$
\begin{aligned}
& \operatorname{pdf}_{w}(z ; \alpha, \beta, \gamma)=\frac{\Gamma\left(\frac{\alpha+\beta+\gamma}{2}\right) a_{2}^{\frac{\alpha+\gamma}{2}}}{\Gamma\left(\frac{\alpha+\beta+2}{2}\right) \Gamma\left(\frac{\gamma}{2}\right) a_{1}^{\frac{\alpha}{2}}\left(1+a_{2}\right)^{\frac{\alpha+\beta+\gamma}{2}}} \\
& \times\left[\frac{\alpha \dot{a}_{1}}{2 a_{1}}{ }_{2} F_{1}\left(\frac{\alpha+\beta+\gamma}{2}, \frac{\alpha+2}{2}, \frac{\alpha+\beta+2}{2} ; \frac{a_{2}\left(a_{2}^{-1}-a_{1}^{-1}\right)}{1+a_{2}}\right)\right. \\
&\left.\quad+\frac{\beta \dot{a}_{2}}{2 a_{2}}{ }_{2} F_{1}\left(\frac{\alpha+\beta+\gamma}{2}, \frac{\alpha}{2}, \frac{\alpha+\beta+2}{2} ; \frac{a_{2}\left(a_{2}^{-1}-a_{1}^{-1}\right)}{1+a_{2}}\right)\right] .
\end{aligned}
$$

The Gaussian hypergeometric functions converge because

$$
0<\frac{a_{2}\left(a_{2}^{-1}-a_{1}^{-1}\right)}{1+a_{2}}<1 .
$$

When $a_{1}<a_{2}$, the same expression applies with $\left(a_{1}, a_{2}\right)$ and $(\alpha, \beta)$ exchanged. In the special case $\gamma=2$ we obtain the very simple result

$$
\operatorname{pdf}_{w}(z ; \alpha, \beta, 2)=\frac{\alpha \frac{\dot{a}_{1}}{1+a_{1}}+\beta \frac{\dot{a}_{2}}{1+a_{2}}}{2\left(1+a_{1}\right)^{\frac{\alpha}{2}}\left(1+a_{2}\right)^{\frac{\beta}{2}}} .
$$

For the case $\gamma=2 s+2$ the formula simplifies to a linear combination of two finite polynomials. This result relies on the following lemma, where, recall, $A_{n_{1}, n_{2}}\left(a_{1}, a_{2}\right)$ denotes the matrix $\operatorname{diag}\left(a_{i} I_{n_{i}}, i=1,2\right)$, and $C_{j}(A)$ denotes the top-order zonal polynomial of degree $j$ of a matrix $A$.

Lemma 4.4. We have

$$
\left(\frac{1}{2}\right)_{j} C_{j}\left(A_{n_{1}, n_{2}}\left(a_{1}, a_{2}\right)\right)=\sum_{k=0}^{j}\left(\begin{array}{l}
j \\
k
\end{array}\right)\left(\frac{n_{1}}{2}\right)_{k}\left(\frac{n_{2}}{2}\right)_{j-k} a_{1}^{k} a_{2}^{j-k} .
$$

Using this result we obtain the simpler form

$$
\begin{aligned}
& \operatorname{pdf}_{w}(z ; \alpha, \beta, 2 s+2)=\frac{\left(\frac{1}{2}\right)_{s}}{2 s !\left(1+a_{1}\right)^{\frac{\alpha}{2}}\left(1+a_{2}\right)^{\frac{\beta}{2}}} \\
& \times\left[\frac{\alpha \dot{a}_{1}}{1+a_{1}} C_{s}\left(A_{\alpha+2, \beta}\left(\varphi_{1}, \varphi_{2}\right)\right)+\frac{\beta \dot{a}_{2}}{1+a_{2}} C_{s}\left(A_{\alpha, \beta+2}\left(\varphi_{1}, \varphi_{2}\right)\right)\right],
\end{aligned}
$$

where $\varphi_{i}:=a_{i} /\left(1+a_{i}\right), i=1,2$. The proof of Proposition 4.3 and its special cases (4.13) and (4.14) is given in Appendix 4 of the Supplement.

Applying Proposition 4.2 to the unbalanced model, we require two applications of the result, one for each component of the cdf. The coefficients and degrees of freedom for each part are given in the following table: 


\begin{tabular}{cccccccc}
\hline Interval & $\alpha$ & $a_{1}$ & $\varphi_{1}$ & $\beta$ & $a_{2}$ & $\varphi_{2}$ & $\gamma$ \\
\hline$-\left(m_{1}-1\right)<z<z_{2}$ & $r$ & $-\frac{1}{\psi_{1}(z, \lambda)}$ & $\frac{1}{1-\psi_{1}(z, \lambda)}$ & $n_{2}$ & $-\frac{\psi_{2}(z, \lambda)}{\psi_{1}(z, \lambda)}$ & $\frac{\psi_{2}(z, \lambda)}{\psi_{2}(z, \lambda)-\psi_{1}(z, \lambda)}$ & $n_{1}$ \\
$z_{2}<z<1$ & $n_{1}$ & $-\psi_{1}(z, \lambda)$ & $-\frac{\psi_{1}(z, \lambda)}{1-\psi_{1}(z, \lambda)}$ & $n_{2}$ & $-\psi_{2}(z, \lambda)$ & $-\frac{\psi_{2}(z, \lambda)}{1-\psi_{2}(z, \lambda)}$ & $r$ \\
\hline
\end{tabular}

In Figure 3 we display the exact density for the case when $r_{1}=r_{2}=1$ (so $r=2$ ) and one of the two groups has fixed size 2, varying the size of the other group, and hence varying $n$. The density is plotted for three different values of $\lambda$. When the model is balanced ( $r=m=2$, so that $n=4)$ the density is analytic on $\Lambda=(-1,1)$. On the other hand, when the model is unbalanced there is a clearly visible point of non-analyticity at $z_{2}$. Using expression (4.3), this point is -.4545 for $n=10$, and it approaches $-1 / 3$ from the left as $n \rightarrow \infty$.
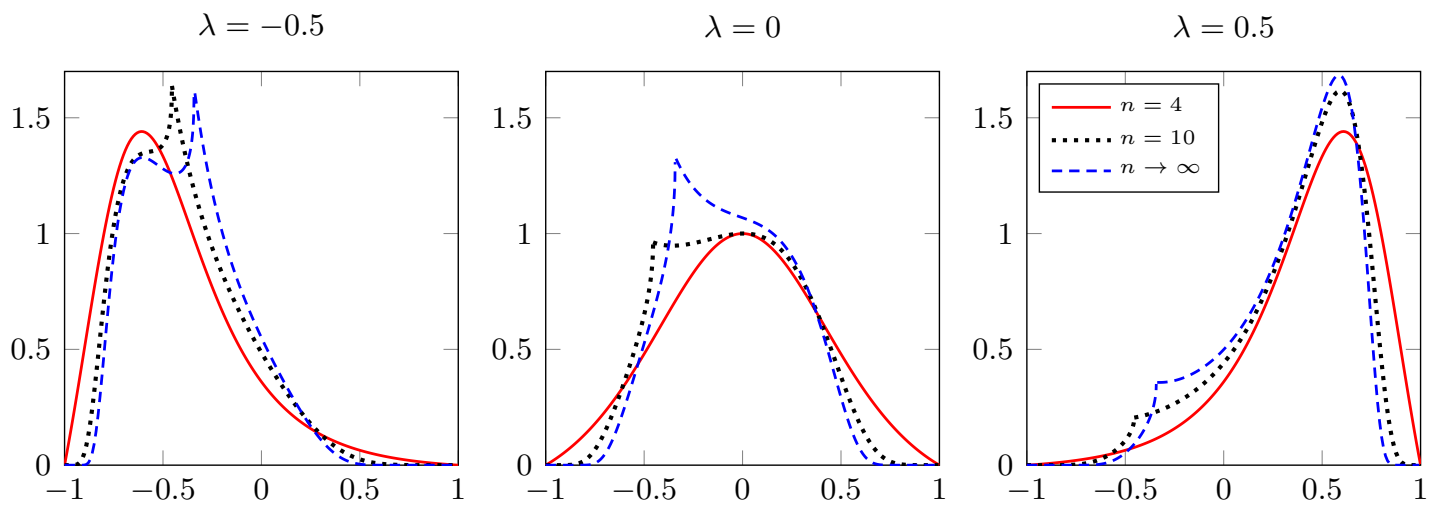

Figure 3: Density of $\hat{\lambda}_{\mathrm{ML}}$ for the Gaussian pure Group Interaction model with two groups, one of which has size $m_{1}=2$.

The plots show clearly that the density has a single component only when the model is balanced. As the difference between $m_{1}$ and $m_{2}$ increases, the difference between the two components becomes more apparent, and the density becomes less smooth at the point $z_{2}$. Incidentally, in this model, the density is continuous at the point of non-analyticity. In other models the density may be unbounded at such a point. One could interpret this phenomenon as a consequence of imposing the same parameter $\lambda$ on the two different groups.

Additional figures for values of $m_{1}>2$ are given in the Supplement. All of these figures show that the properties of $\hat{\lambda}_{\mathrm{ML}}$ are, in this model with just two groups, almost invariant to the sample size, a property related to, but not implied by the asymptotic properties for a fixed number of groups mentioned earlier. However, even though the estimator is 
not consistent under some asymptotic regimes, there is certainly no evidence here that suggests not using maximum likelihood in this model.

\subsection{Probability of Underestimation: the Median}

We next consider the special case of equation (4.1) with $z=\lambda$, so that the object of interest becomes $\operatorname{Pr}\left(\hat{\lambda}_{\mathrm{ML}} \leq \lambda ; \lambda\right)$, the probability of underestimating $\lambda$. This seems to be the only available method for examining the median bias of $\hat{\lambda}_{\mathrm{ML}}$ in this unbalanced model. When $z=\lambda$, we have $c_{i}(\lambda, \lambda)=1$ for all $i$ and all $\lambda$, so that

$$
\operatorname{Pr}\left(\hat{\lambda}_{\mathrm{ML}} \leq \lambda ; \lambda\right)=\operatorname{Pr}\left(\sum_{i=1}^{N}\left(g_{i}(\lambda)-\bar{g}(\lambda)\right) \chi_{n_{i}}^{2} \leq 0\right) .
$$

If $\lambda \geq z_{p}$, which includes all values $\lambda \geq 0$, all of the coefficients in this expression are negative, except the last. Thus, for $\lambda \geq z_{p}$ we have

$$
\operatorname{Pr}\left(\hat{\lambda}_{\mathrm{ML}} \leq \lambda ; \lambda\right)=\operatorname{Pr}\left(\chi_{r}^{2} \leq \sum_{i=1}^{p} \psi_{i}(\lambda) \chi_{n_{i}}^{2}\right),
$$

where

$$
\psi_{i}(\lambda):=\psi_{i}(\lambda, \lambda)=-\frac{g_{i}(\lambda)-\bar{g}(\lambda)}{g_{p+1}(\lambda)-\bar{g}(\lambda)}, i=1, . ., p .
$$

An exact formula can be derived from equation (4.16) by using the results discussed above, but the result is too complex to be useful. A simpler, more helpful approach, is to use the Fisher approximation for the linear combination on the right, i.e., to assume

$$
\sum_{i=1}^{p} \psi_{i}(\lambda) \chi_{n_{i}}^{2} \cong \alpha \chi_{v}^{2}
$$

where $\alpha=\pi_{2} / \pi_{1}$, and $v(\lambda):=\pi_{1}^{2} / \pi_{2}$, and $\cong$ denotes equality in distribution. In this case things simplify greatly, because $\pi_{1}=r$, so $\alpha=\pi_{2} / r, v(\lambda)=r^{2} / \pi_{2}$, which produces the approximation, for $\lambda \geq z_{p}$,

$$
\operatorname{Pr}\left(\hat{\lambda}_{\mathrm{ML}} \leq \lambda ; \lambda\right) \simeq \operatorname{Pr}\left(\chi_{r}^{2} \leq \alpha \chi_{v(\lambda)}^{2}\right)=\operatorname{Pr}\left(\mathrm{F}_{r, v(\lambda)} \leq \frac{\alpha v(\lambda)}{r}\right)=\operatorname{Pr}\left(\mathrm{F}_{r, v(\lambda)} \leq 1\right),
$$

an analogue of the result given earlier for the balanced model. But, as we have noted earlier, $\operatorname{Pr}\left(F_{r, v} \leq 1\right)>.5$ if $v>r$, and vice versa. That is, up to the accuracy of this approximation, $\operatorname{med}\left(\hat{\lambda}_{\mathrm{ML}}\right)<\lambda$ if $v(\lambda)>r$, and $\operatorname{med}\left(\hat{\lambda}_{\mathrm{ML}}\right)>\lambda$ if $v(\lambda)<r$. There is therefore a negative median-bias when $\lambda$ is in the set $\left\{\lambda: \lambda>z_{p}, v(\lambda)>r\right\}$, and a positive median-bias when $\lambda \in\left\{\lambda: \lambda>z_{p}, v(\lambda)<r\right\}$. 
For the interval $-\left(m_{1}-1\right)<\lambda<z_{2}$, the opposite situation occurs: all coefficients in the linear combination are positive, except the first. Thus an analogous expression, and an analogous approximation, can be deduced for $\lambda$ in this region. For values of $\lambda$ between $z_{2}$ and $z_{p}$ the expression for $\operatorname{Pr}\left(\hat{\lambda}_{\mathrm{ML}} \leq \lambda ; \lambda\right)$ will involve the difference between two positive linear combinations of $\chi^{2}$ variates. Each can separately be approximated as above, and an approximation for the probability easily obtained. For each interval the approximation takes the form, in obvious notation,

$$
\operatorname{Pr}\left(\hat{\lambda}_{\mathrm{ML}} \leq \lambda ; \lambda\right) \simeq \operatorname{Pr}\left(\mathrm{F}_{v_{L}, v_{R}} \leq 1\right)
$$

so the only things needed are the pairs $\left(v_{L}, v_{R}\right)$ appropriate to each interval. The reason for this is as follows: when the approximation is used for both sides of an inequality we have, symbolically,

$$
\begin{aligned}
\operatorname{Pr}\left(\alpha_{L} \chi_{v_{L}}^{2} \leq \alpha_{R} \chi_{v_{R}}^{2}\right) & =\operatorname{Pr}\left(\mathrm{F}_{v_{L}, v_{R}} \leq \frac{v_{R} \alpha_{R}}{v_{L} \alpha_{L}}\right) \\
& =\operatorname{Pr}\left(\mathrm{F}_{v_{L}, v_{R}} \leq-\frac{\pi_{1 R}}{\pi_{1 L}}\right) \\
& =\operatorname{Pr}\left(\mathrm{F}_{v_{L}, v_{R}} \leq 1\right),
\end{aligned}
$$

since it is always the case that $\pi_{1 R}+\pi_{1 L}=0$. This is an analogue of the corresponding result for the balanced model given earlier. For example, in the case $p=4$ we have four intervals to accommodate, and the following results for the approximation to $\operatorname{Pr}\left(\hat{\lambda}_{\mathrm{ML}} \leq \lambda ; \lambda\right)$ are typical of the general case:

$$
\begin{array}{rll}
-\left(m_{1}-1\right)<\lambda<z_{2}: & \operatorname{Pr}\left(\mathrm{F}_{v_{A}, n_{1}} \leq 1\right), & v_{A}:=\frac{\left(n_{1} \psi_{1}\right)^{2}}{\left(r+\sum_{i=2}^{4} n_{i} \psi_{i}^{2}\right)} \\
z_{2}<\lambda<z_{3}: & \operatorname{Pr}\left(\mathrm{F}_{v_{B R}, v_{B L}} \leq 1\right), & v_{B R}:=\frac{\left(n_{1} \psi_{1}+n_{2} \psi_{2}\right)^{2}}{n_{1} \psi_{1}^{2}+n_{2} \psi_{2}^{2}}, v_{B L}:=\frac{\left(n_{1} \psi_{1}+n_{2} \psi_{2}\right)^{2}}{\left(n_{3} \psi_{3}^{2}+n_{4} \psi_{4}^{2}+r\right)} \\
z_{3}<\lambda<z_{4}: & \operatorname{Pr}\left(\mathrm{F}_{v_{C R}, v_{C L}} \leq 1\right), & v_{C R}:=\frac{\left(n_{4} \psi_{4}+r\right)^{2}}{\sum_{i=1}^{3} n_{i} \psi_{i}^{2}}, v_{C L}:=\frac{\left(n_{4} \psi_{4}+r\right)^{2}}{n_{4} \psi_{4}^{2}+r} \\
z_{4}<\lambda<1: & \operatorname{Pr}\left(\mathrm{F}_{r, v_{D}} \leq 1\right), & v_{D}:=\frac{r^{2}}{\sum_{i=1}^{4} n_{i} \psi_{i}^{2}} .
\end{array}
$$

Evidence on the accuracy of the approximation is given in the following table, where we compare exact results (obtained by simulating (4.15)) with those obtained by the approximation, for the case $p=4$, and three different combinations of the group sizes (design 1: $m_{1}=5, m_{2}=10, m_{3}=15, m_{4}=20$; design 2: $m_{1}=10, m_{2}=20, m_{3}=$ 30, $m_{4}=40$; design 3: $\left.m_{1}=5, m_{2}=50, m_{3}=100, m_{4}=150\right)$. 


\begin{tabular}{ccccccc}
\hline & \multicolumn{2}{c}{$\lambda=-.9$} & \multicolumn{2}{c}{$\lambda=0$} & \multicolumn{2}{c}{$\lambda=.9$} \\
\cline { 2 - 7 } Design & Exact & Approx. & Exact & Approx. & Exact & Approx. \\
1 & .561 & .561 & .580 & .579 & .582 & .583 \\
2 & .581 & .580 & .587 & .587 & .588 & .589 \\
3 & .553 & .553 & .585 & .585 & .592 & .592 \\
\hline
\end{tabular}

Note that for all cases considered in the table, $\operatorname{Pr}\left(\hat{\lambda}_{\mathrm{ML}} \leq \lambda ; \lambda\right)>.5$, i.e., the median bias is negative. Based on our calculations using the approximation developed in this section, this seems a general result for whenever $\lambda \in\left(z_{p}, 1\right)$ (similarly, the median bias seems to be always positive for $\left.\lambda \in\left(-\left(m_{1}-1\right), z_{2}\right)\right)$.

\subsubsection{Probability of Underestimation: Two Group Sizes}

In the case of two distinct group sizes $(p=2)$ the two intervals $-\left(m_{1}-1\right)<\lambda<z_{2}$, and $z_{2}<\lambda<1$ make up all of $\Lambda$, and each of the above expressions involves a positive linear combination of just two $\chi^{2}$ variates. We can therefore use the result in Proposition 4.1 to obtain expressions for the required probability in each of these intervals. For the first (upper) interval, $\phi=1 / \psi_{1}(\lambda)$, and $\phi A=\operatorname{diag}\left(I_{n_{1}},\left(\psi_{2}(\lambda) / \psi_{1}(\lambda)\right) I_{n_{2}}\right)$, and we obtain

$$
\begin{aligned}
\operatorname{Pr}\left(\hat{\lambda}_{\mathrm{ML}} \leq \lambda ; \lambda\right)=\left(\frac{\psi_{2}(\lambda)}{\psi_{1}(\lambda)}\right)^{-\frac{n_{2}}{2}} \sum_{j=0}^{\infty} \frac{\left(\frac{n_{2}}{2}\right)_{j}}{j !}\left(1-\frac{\psi_{1}(\lambda)}{\psi_{2}(\lambda)}\right)^{j} \\
\times \operatorname{Pr}\left(\operatorname{Beta}\left(j+\frac{n_{1}+n_{2}}{2}, \frac{r}{2}\right) \leq \frac{1}{1+\psi_{1}(\lambda)}\right)
\end{aligned}
$$

For the lower interval, $\phi=1 / \tilde{\psi}_{2}(\lambda)$ and $\phi A=\operatorname{diag}\left(I_{n_{2}},\left(\tilde{\psi}_{3}(\lambda) / \tilde{\psi}_{2}(\lambda)\right) I_{r}\right)$, so that

$$
\begin{aligned}
\operatorname{Pr}\left(\hat{\lambda}_{\mathrm{ML}} \leq \lambda ; \lambda\right)=1-\left(\frac{\tilde{\psi}_{3}(\lambda)}{\tilde{\psi}_{2}(\lambda)}\right)^{-\frac{r}{2}} & \sum_{j=0}^{\infty} \frac{\left(\frac{r}{2}\right)_{j}}{j !}\left(1-\frac{\tilde{\psi}_{2}(\lambda)}{\tilde{\psi}_{3}(\lambda)}\right)^{j} \\
& \times \operatorname{Pr}\left(\operatorname{Beta}\left(j+\frac{n_{2}+r}{2}, \frac{n_{1}}{2}\right) \leq \frac{1}{1+\tilde{\psi}_{2}(\lambda)}\right) .
\end{aligned}
$$

These formulae can be used to plot the probability $\operatorname{Pr}\left(\hat{\lambda}_{\mathrm{ML}} \leq \lambda ; \lambda\right)$ as a function of $\lambda$. Figure 10 in the Supplement plots (a truncated version of) the formulae (4.17) and (4.18) in the case of two group sizes, for $\lambda \in(-1,1)$, and for a variety of values of $r_{1}, r_{2}, m_{1}, m_{2}$. The results were compared to simulation results, and also to the Fisher approximation discussed above. All three methods give virtually identical results. As $r_{1}$ and $r_{2}$ increase the probability of underestimation converges to .5 , but the probability of underestimation can be very sensitive to $\lambda$, even for values of $\lambda$ in $(-1,1)$. 


\subsection{Approximating the Distribution}

The approach used above to approximate $\operatorname{Pr}\left(\hat{\lambda}_{\mathrm{ML}} \leq \lambda ; \lambda\right)$ can be applied to the expressions for the cdf itself, in each interval of its domain. Considering just the case $p=2$, we simply need to replace $\psi_{1}$ and $\psi_{2}$ by $\psi_{1}(z, \lambda)$ and $\psi_{2}(z, \lambda)$ in the definitions of $\pi_{1}, \pi_{2}$, although, in the case of the distribution function the results are not quite so simple as those given above for $\operatorname{Pr}\left(\hat{\lambda}_{\mathrm{ML}} \leq \lambda ; \lambda\right)$. The relevant expressions for the cdf are, in the case $p=2$,

$$
\operatorname{Pr}\left(\hat{\lambda}_{\mathrm{ML}} \leq z ; \lambda\right) \simeq \operatorname{Pr}\left(\mathrm{F}_{v_{1}(z, \lambda), n_{1}} \leq u_{1}(z, \lambda)\right),
$$

for $\lambda<z_{2}$, and

$$
\operatorname{Pr}\left(\hat{\lambda}_{\mathrm{ML}} \leq z ; \lambda\right) \simeq \operatorname{Pr}\left(\mathrm{F}_{r, v_{2}(z, \lambda)} \leq u_{2}(z, \lambda)\right)
$$

for $\lambda>z_{2}$, where

$$
u_{1}(z, \lambda):=-\frac{n_{1} \psi_{1}(z, \lambda)}{n_{2} \psi_{2}(z, \lambda)+r}, v_{1}(z, \lambda):=\frac{\left(n_{2} \psi_{2}(z, \lambda)+r\right)^{2}}{n_{2} \psi_{2}^{2}(z, \lambda)+r},
$$

and

$$
u_{2}(z, \lambda):=\frac{n_{1} \psi_{1}(z, \lambda)+n_{2} \psi_{2}(z, \lambda)}{n_{1} \psi_{1}(z, \lambda)+n_{2} \psi_{2}(z, \lambda)}, v_{2}(z, \lambda):=\frac{\left(n_{1} \psi_{1}(z, \lambda)+n_{2} \psi_{2}(z, \lambda)\right)^{2}}{n_{1} \psi_{1}^{2}(z, \lambda)+n_{2} \psi_{2}^{2}(z, \lambda)} .
$$

Analytic differentiation to obtain the density is messy, but easily accomplished by a symbolic mathematical package, and again can be extended to cases with $p>2$ without difficulty.

\subsection{Group-Specific Regressions}

We now consider generalizations to the pure unbalanced Group Interaction model with regressors. Compared to the balanced case, unbalancedness has the favorable consequence that group fixed effects do not render inference on the full parameter impossible. ${ }^{19}$

Similarly to Section 3.6.2, we focus on the case in which all $\beta$ coefficients are group specific. We show that in this case the cdf of $\hat{\lambda}_{\mathrm{ML}}$ admits a very simple representation when group fixed effects are present, regardless of the values of the regressors. Within each group the model is a balanced Group Interaction model, or, stacking groups of same size,

$$
y_{i}=\lambda\left(I_{r_{i}} \otimes B_{m_{i}}\right) y_{i}+\bigoplus_{j=1}^{r_{i}} X_{i j} \beta_{i j}+\varepsilon_{i}, i=1, . ., p
$$

\footnotetext{
${ }^{19}$ The fixed effects matrix $\bigoplus_{i=1}^{p}\left(I_{r_{i}} \otimes \iota_{m_{i}}\right)$ span an eigenspace of $W$ both in the balanced $(p=1)$ and in the unbalanced case $(p>1)$. However, when $p>1$, the presence of fixed effects, does not imply the same degeneracy that occurs when $p=1$. This is a consequence of the fact that $W$ has more than two eigenspaces when $p>1$.
} 
where $y_{i}$ is $r_{i} m_{i} \times 1, X_{i j}$ is an $m_{i} \times k_{i j}$ matrix containing a column of ones (with $k_{i j} \leq m_{i}$ ), and $\beta_{i}$ is $\sum_{j=1}^{r_{i}} k_{i j} \times 1$ (that is, for each of the $p$ distinct group sizes, the model is a balanced model with group specific regressors). This corresponds to an unbalanced Group Interaction model with $X=\bigoplus_{i=1}^{p} \bigoplus_{j=1}^{r_{i}} X_{i j}, k=\sum_{i=1}^{p} \sum_{j=1}^{r_{i}} k_{i j}$, and $\beta^{\prime}=\left(\beta_{11}^{\prime}, . ., \beta_{1 r_{1}}^{\prime}, \ldots, \beta_{p 1}^{\prime}, . ., \beta_{p r_{p}}^{\prime}\right)$. By Lemma B.3 in the Supplement, if the model contains group fixed effects, then $\operatorname{col}(X)$ is spanned by $k$ eigenvectors of $W=\operatorname{diag}\left(I_{r_{i}} \otimes B_{m_{i}}, i=\right.$ $1, \ldots, p)$. Then, provided only that $\operatorname{col}(X)$ does not contain all eigenvectors of $W$ associated with eigenvalues other than $\omega$ (to avoid degeneracy of the score), by the same argument as in Section 3.6.2 we obtain $\operatorname{Pr}\left(\hat{\lambda}_{\mathrm{ML}} \leq z ; \lambda\right)=\operatorname{Pr}\left(\sum_{i=1}^{p} d_{i i}(z, \lambda) \chi_{n_{i}-n_{i}(X)}^{2} \leq 0\right)$, where the $\chi_{n_{i}-n_{i}(X)}^{2}$ variates are independent, $n_{i}(X):=\operatorname{dim}\left(\operatorname{col}(X) \cap \operatorname{col}\left(I_{r} \otimes L_{m_{i}}\right)\right)$, and we use the convention that $\chi_{0}^{2}=0$. Using the definition (2.3) of the coefficients $d_{i i}(z, \lambda)$, we have

$$
\operatorname{Pr}\left(\hat{\lambda}_{\mathrm{ML}} \leq z ; \lambda\right)=\operatorname{Pr}\left(\sum_{i=1}^{p}\left(g_{i}(z)-\bar{g}(z)\right)\left(\frac{z+m_{i}-1}{\lambda+m_{i}-1}\right)^{2} \chi_{n_{i}-n_{i}(X)}^{2} \leq 0\right),
$$

where the coefficients $g_{i}(z)-\bar{g}(z)$ are given in equation (4.2). Representation (4.19) reveals an unexpected property of $\hat{\lambda}_{\mathrm{ML}}$. Specifically, recalling from Section 4.1 that $g_{i}(z)-\bar{g}(z)<0$ for any $i=1, \ldots, p$ and for any $z \in\left(z_{p}, 1\right)$, where $z_{p}$ is the point at which the coefficient $g_{p}(z)-\bar{g}(z)$ changes sign, representation (4.19) implies that $\operatorname{Pr}\left(\hat{\lambda}_{\mathrm{ML}} \leq z ; \lambda\right)=1$ for any $z>z_{p}$. That is, for this model the support of the distribution of $\hat{\lambda}_{\mathrm{ML}}$ is not the entire $\Lambda$, but its subset $\left(-\left(m_{1}-1\right), z_{p}\right)$. That is, the probability that $\hat{\lambda}_{\mathrm{ML}}$ is in $\left(z_{p}, 1\right)$ is 0 even if the true value of $\lambda$ is in $\left(z_{p}, 1\right)$.

Similarly to what was done in Section 3.6.2, one can study the distribution of $\hat{\lambda}_{\mathrm{ML}}$ under different asymptotic regimes, but we omit these calculations for the sake of brevity.

\section{Concluding Remarks}

In Hillier and Martellosio (2016) we presented a general result, equation (2.1) above, giving an exact representation for the distribution function of the quasi-maximum likelihood estimator for the autoregressive parameter $\lambda$ in the spatial autoregressive model (1.3), valid for any distribution of $\varepsilon$. Some examples of the application of the result to particular cases were given in $\mathrm{H} \& \mathrm{M}$, but the earlier paper concentrated mainly on its more general consequences. In the present paper we have explored the application of the result to a particular class of models - those based on spatial weights matrices that embody group-interaction. These models are important in various areas of application to the study of networks, and to panels with a spatial autoregressive component. Starting from equation (2.1) we have 
been able to present a very complete set of results for likelihood-based inference in the pure balanced Group Interaction model under mixed-Gaussian assumptions. We have also been able to generalize these simple results to some special cases of models involving regressors, for example, models with a common mean across all observations, and models with group-specific regressors satisfying certain assumptions.

The pure balanced model is possibly the simplest example of equation (1.3) one can imagine, and the ability to carry out the above program is due to the fact that this model is a regular exponential family. We have then discussed the much more realistic unbalanced model, a model that is considerably more difficult. Again, that is no doubt because the unbalanced model is not a regular exponential family, but a curved exponential family in which the dimension of the sufficient statistic is larger than that of the parameter space. Exact results in this model are available in closed form, but are very complex. Thus, in addition to reporting the exact results, we have given some approximations that appear to work well, and which generalize nicely the simpler result for the balanced model. There is more work to be done on the unbalanced model however.

Finally, it should be remarked that there are alternatives to the QMLE estimator considered in this paper that have been proposed in the literature, but it seems unlikely that the methods used here would be available for such alternatives. For example, GMM estimators have been proposed for this model (see, e.g., Lee and Liu (2010) and references therein), and this proposal could be extended to the class of generalized empirical likelihood/GMM estimators introduced in the influential paper by Smith (1997).

\section{References}

Andrews, D.W.K. (1993) Exactly median-unbiased estimation of first-order autoregressive/unit root models, Econometrica 61, 139-165.

Arnold, S.F. (1979) Linear models with exchangeably distributed errors, Journal of the American Statistical Association 74, 194-199.

Baltagi, B.H. (2006) Random effects and spatial autocorrelation with equal weights, Econometric Theory 22, 973-984.

Bao, Y. (2013) Finite-sample bias of the QMLE in spatial autoregressive models, Econometric Theory 29, 68-88.

Barndorff-Nielsen, O. (1980) Conditionality resolutions, Biometrika 67, 293-310. 
Buckley, M.J., and Eagleson, G.K. (1988) An approximation to the distribution of quadratic forms in normal random variables, Australian Journal of Statistics 30A, 150-159.

Bramoullé, Y., Djebbari H., and Fortin B. (2009), Identification of peer effects through social networks, Journal of Econometrics 150, 41-55.

Case, A. (1992) Neighborhood influence and technological change, Regional Science and Urban Economics 22, 491-508.

Daniels, H.E. (1987) Tail probability approximations, International Statistical Review 55, $37-48$.

Davezies, L., D'Haultfoeuille, X., and Fougère, D. (2009) Identification of peer effects using group size variation. Econometrics Journal 12, 397-413.

de Paula (2016) Econometrics of network models, Cemmap working paper CWP06/16.

Hall, P., (1983) Chi squared approximations to the distribution of a sum of independent random variables, The Annals of Probability 11, 4, 1028-1036.

Hillier, G.H. and Martellosio, F. (2016) Exact and higher-order properties of the MLE in spatial autoregressive models, with applications to inference. Manuscript (This is a substantially revised version of Hillier, G.H. and Martellosio, F. (2013), Properties of the maximum likelihood estimator in spatial autoregressive models. Cemmap Working Papers, CWP44/13.)

Hsieh, C.-S., Lee, L.F. (2016) A social interactions model with endogenous friendship formation and selectivity, Journal of Applied Econometrics 31, 301-319.

James, A.T. (1964) Distributions of matrix variates and latent roots derived from normal samples, Annals of Mathematical Statistics 35, 475-501.

Johnson, N.L., Kotz, S. and Balakrishnan, N. (1994) Distributions in Statistics-Continuous Univariate Distributions, Vol. 1 (Second Edition). New York: John Wiley \& Sons.

Kelejian, H.H., Prucha, I.R. and Yuzefovich, Y. (2006) Estimation problems in models with spatial weighting matrices which have blocks of equal elements. Journal of Regional Science 46, 507-515.

King, M.L. (1981) A small sample property of the Cliff-Ord test for spatial autocorrelation. Journal of the Royal Statistical Society B 43, 263-4.

Lee, L.F. (2004) Asymptotic distributions of quasi-maximum likelihood estimators for spatial autoregressive models, Econometrica 72, 1899-1925. 
Lee, L.F. (2007) Identification and estimation of econometric models with group interactions, contextual factors and fixed effects, Journal of Econometrics 140, 333-374.

Lee, L.F. and Liu, X. (2010) Efficient GMM estimation of high order spatial autoregressive models with autoregressive disturbances, Econometric Theory 26, 187-230.

Lee, L.F., Liu, X., Lin, X. (2010) Specification and estimation of social interaction models with network structures, The Econometrics Journal 13, 145-176.

Lee, L-F. and Yu, J. (2010) Estimation of spatial autoregressive panel data models with fixed effects, Journal of Econometrics 154, 165-185.

Lugannani, R. and Rice, S.O. (1980) Saddlepoint approximations for the distribution of the sum of independent random variables, Advances in Applied Probability. 12, 475-490.

Manski, C.F. (1993) Identification of endogenous social effects: the reflection problem, The Review of Economic Studies 60, 531-542.

Moffitt, R. (2001) Policy interventions, low-level equilibria and social interactions, in Social Dynamics, ed. by S. Durlauf, and P. Young. MIT Press.

Robinson, P.M., Rossi, F. (2015a) Refinements in maximum likelihood inference on spatial autocorrelation in panel data, Journal of Econometrics 189, 447-456.

Robinson, P.M., Rossi, F. (2015b) Refined tests for spatial correlation, Econometric Theory $31,1249-1280$.

Ruben, H. (1962) Probability content of regions under spherical normal distributions, IV: the distribution of homogeneous and non-homogeneous quadratic functions of normal variables, The Annals of Mathematical Statistics 33, 542-570.

Smith, R.J. (1997) Alternative semi-parametric likelihood approaches to generalised method of moments estimation, The Economic Journal, 107, 503-519. 